\title{
Abstraction-Carrying Code: a Model for Mobile Code Safety
}

\author{
Elvira ALBERT \\ DSIC. Complutense University of Madrid \\ Avda. Complutense s/n. E-28040 Madrid. SPAIN \\ Germán PUEBLA \\ Technical University of Madrid (UPM) \\ E-28660 Boadilla del Monte. Madrid. SPAIN \\ Manuel HERMENEGILDO \\ Technical University of Madrid, Spain and \\ CS/EECE Depts, University of New Mexico, USA
}

\begin{abstract}
Proof-Carrying Code (PCC) is a general approach to mobile code safety in which programs are angmented with a certificate! (or proof). The intended benefit is that the program consumer can locally validate the certificate w.r.t. the "untrusted" program by means of a certificate checker-a process which should be much simpler, efficient, and automat ic than generating the original proof. The practical uptake of PCC greatly depends on the existence of a variety of enabling technologies which allow both proving programs correct and replacing a costly verification process by an efticient checking procedure on the consumer side. In this work we propose AbstractionCarrying Code (ACC), a novel approach which uses abstract interpretation as enabling technology. We argue that the large body of applications of abstract interpretation to program verification is amenable to the overall $\mathrm{PCC}$ scheme. In particular, we rely on an expressive class of safety policies which can be defined over different abstract domains. We use an abstraction (or abstract model) of the program computed by standard static analyzers as a certificate. The validity of the abstraction on the consumer sirle is chceked in a single pass by a very efficient and specialized abstract-interpreter. We believe that ACC brings the expressiveness, flexibility and automation which is inherent in abstract interpretation techniques to the area of molsile code safety.
\end{abstract}

Keywords: Logic Programming. Static Analysis. Abstract Interpretation. 


\section{$\S 1$ Introduction}

One of the most important challenges which computing research faces today is the development of security techniques for verifying that the execution of a program (possibly) supplied by an untrusted source is safe, i.e., that it meets certain properties according to a predefined safety policy. Proof-Carrying Code $(\mathrm{PCC})^{34}$ is a general framework for mobile code safety which proposes to associate safety information in the form of a certificate to programs. The certificate (or proof) is created at compile time, and packaged along with the code. The consumer who receives or downloads the code+certificate package can then run a checker which by an efficient inspection of the corle and the certificate, can verify the validity of the certificate and thus conmpliance with the safety policy. The key benefit of this "certificate-based" appronch to mobile code safety is that the consumer's task is reduced from the level of proving to the level of checking, a task that should be much simpler. efficient, and automatic than generating the original certificate.

The practical uptake of PCC greatly depends on the existence of a variety of enabling technologies which allow:

1. defining expressive safety policies covering a wide range of properties,

2. solving the problem of how to automatically generate the certificates (i.e., automatically proving the programs correct), and

3. replacing a costly verification process by an efficient checking procedure on the consumer side.

The main approaches applied up to now are based on either theoren proving or type analysis. For instance, in PCC the certificate is originally ${ }^{341}$ a proof in firstorder logic of certain uerification conditions and the checking process involves ensiring that the certificate is indeed a valid first-order proof. $\lambda$ Prolog is used ${ }^{3)}$ to define a representation of lemmas and definitions which helps keep the proofs small. Another proposal ${ }^{\text {fi }}$ uses temporal logic to specify security policies in PCC. In Typed Assembly Languages, ${ }^{3(1)}$ the certificate is a type annotation of the assembly language program and the checking process involves a form of type checking. Each of the different approaches possesses their own set of stronger and weaker points. Depending on the particular safety property and the available computing resources in the consumer, some approaches are more suitable than others. In some cases the priority is to reduce the size of the certificate as much as possible in order to fit in small devices or to cope with searce network access (as in, e.g., Oracle-based $\mathrm{PCC}^{(6)}$ or Tactic-based $\mathrm{PCC}^{41}$ ), whereas in other cases the priority is to reduce the checking time (as in. e.g., standard $\mathrm{PCC}^{34}$ or lightweight bytecode verification ${ }^{201}$ ). As a result of all this, a successful certificate infrastructure should have a wide set of enabling technologits available for the different requirements.

In this work we propose Abstraction-Carrying Code (ACC), a novel ap- 


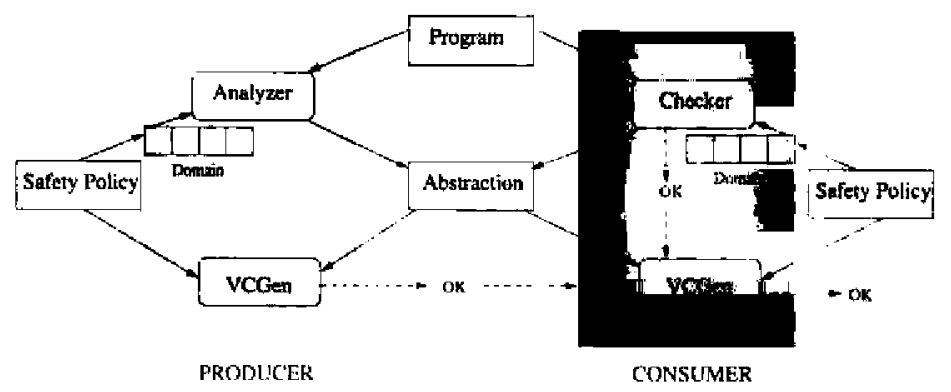

Fig. 1 Abstraction-Carrying Code

proach which uses abstract interprefation ${ }^{13)}$ as enabling techology to handle the difficult practical challenges mentioned above. Abstract interpretation is now a well-established technique which has allowed the development of very sophisticated global static program analyses that are at the same time automatic. provably correct, and practical. The basic idea of abstract interpretation is to infer information on programs by interpreting ("ruming") them nsing abstract values rather than concrete ones. thus obtaining safe approximations of the beliavior of the program. The technique allows inferring a wide range of properties about prograns. Our proposal. ACC, opens the door to the applicability of this inference power as enabling technology for PCC. Figure 1 presents an overview of ACC. The certification process carried out by the code producer is depicted to the left of the figure while the checking process performod by the code consumer appears to the right. In particular, $\mathrm{ACC}$ has the following fundamental elements which can handle the three aforementioned challenges:

1. The first element, common to both producer and consumers. is the Safety Policy. We rely on an expressive class of safety policies based on "abstract"-i.e. symbolic-properties over different abstract domains. Our framework is parametric w.r.t. the abstract domain(s) of interest. which gives us generality and expressiveness. sirce it allows a single concrete implementation of the approach to be used without change for generating certificates for different classes of properties by simply adding domains as "plugitss."

2. The next element at the producer's side is a fixed point-based static Analyzer which automatically infers an alsstract mode] (or simply abstraction) of the mobile code which can then be used to prove that this code is safe w.r.t. the given policy in a straightforward way. In particular, we identify a subset of the analysis results which is sufficient for this purpose.

3. The verification condition generator. VCGen in the figure, generates, from the initial safety policy and the abstraction, a Verification Condation (VC), which can be proved only if the execution of the corle does not violate the safety policy. As in standard PCC methods, this process is performed also by the consumers in order to have a trustworthy VC.

4. Finally, a simple, easy-to-trust (audysis) checker at the consumer's side 
verifies the validity of the information on the mobile coche. It is indeed a specialized abstract interpreter whose key characteristic is that it does not need to iterate in order to reacl a fixed point (in contrast to standard analyzers).

While $\mathrm{ACC}$ is a general approach, for concreteness we develop herein an incarnation of it in the context of (Constraint) Logic Programming, (C)LP, because this paradigm offers a good number of advantages, an important one being the maturity and sophistication of the analysis tools available for it. In particular. a wide range of analysis domains have been developed to infer properties such as data structure shape (with pointer sharing), bounds on data structure sizes, and other operational variable instantiation properties, as well as procedure-level properties such as determinacy, termination, non-failure, and bounds on resource consumption, such as time or space cost (see, e.g., ${ }^{2 !)}$ and its references).

Also for concreteness, we build on the algorithms of (and report on an implementation on) CiaoPP, ${ }^{21}$ the abstract interpretation-based preprocessor of the Ciao multi-paradign CLP system. " CiaoPP uses modular, incremental abstract interpretation as a fundamental tool to obtain information about programs. In CiaoPP, the semantic approximations thts produced have been applied to perform light- aud low-level optimizations during program compilation, including transformations such as multiple abstract specialization, parallelization, partial evaluation, resource usage control, and program verification. More recently, novel and promising applications of such semantic approximations are being applied in the more general context of program development. We report. on our extension of CiaoPP to incorporate $A C C$ and on how this instantiation of ACC already shows promising results.

The article is organized as follows. Section 2 introduces some notation and preliminary notions on CLP and abstract interpretation. In Section 3, we present a general view of ACC. Section 4 describes the assertion language which is used to define our safety policy. Section 5 discusses the generation of program abstractions. In Section 6, we present the verification condition generator which attests compliance of the abstraction with respect to the safety policy. In Section 7, we introduce an abstract interpretation-based checker which validates the safety certificate in the consumer. Section 8 reports on some experiments performed in the CiaopP-based implementation. Finally. Section 9 discusses the work presented in this article and related work.

\section{$\S 2$ Preliminaries}

We assume familiarity with constraint logic programming ${ }^{24)}$ (CLP) and the concepts of abstract interpretation ${ }^{(6)}$ which underlie most analyses in CLP. The remaining of this section introduces some notation and recalls preliminary concepts on these topics.

\subsection{Constraint Logic Programming}

Terms are constructed from variables (e.g., $X$ ) and functors (e.g., $f$ ). We denote by $\left\{X_{1} \mapsto t_{1}, \ldots, X_{n} \mapsto t_{n}\right\}$ the substitution $\sigma$ with $\sigma\left(X_{i}\right)=t_{i}$ for all 
$i=1 \ldots \ldots n$ with $X_{i} \neq X_{j}$ if $i \neq j$ and $\sigma(X)=X$ for any other variable $X$. where $t_{i}$ are terms. The identity substitution, which we denote by $i d$ is such that $\forall X i d(X)=X$. A renaming is a substitntion $\rho$ for which there exists the inverse $\rho^{-1}$ such that $\rho \rho^{-1} \equiv \rho^{-1} \rho \equiv i d$. We say that a renaming $\rho$ is a renaming substitution of term $t_{1}$ w.r.t. term $t_{2}$ if $t_{2}=\rho\left(t_{1}\right)$.

A literal has the form $p\left(t_{1}, \ldots, t_{n}\right)$ where $p / n$ is a procedure name (predicate symbol), $n$ is its arity and $t_{i}$ are terms. Most real-life (C)LP programs use procedures which are not defined in the program (modulc) being developed. Thus, procedures are classified into internal and extermal. Internal procedures are defined in the current program (module), whereas external procedures are not. Examples of external procedures include the traditional "built-in" (predefined) procedures, such as constraints. basir input/output facilities (e.g., open). We will also consider as external procedures those defined in a different module, procedures written in another language, etc. We assume the existence of a boolean function external s.t. external $\left(p\left(t_{1}, \ldots, t_{n}\right)\right)$ succeeds iff the procedure $p / n$ is external. A goal is a finite sequence of literals. A rule is of the form $H:-B$ where $H$, the head, is a literal and $B$, the body, is a possibly empty finite sequence of literals. A CLP program, or program. is a finite set of rules.

\section{Example 2.1 (Running Example)}

The main procedure, create streams/2, of the following CLP program receives a list of numbers which correspond to certain file names, and returns in the second argument the list of file handlers (streams) associated to the (opened) files:

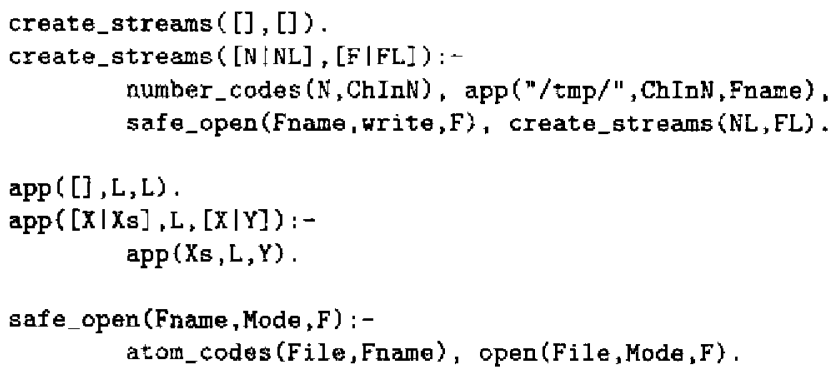

It defines the well-known list concatenation procedure app/3 and uses the following external predicates. The call number codes $(\mathrm{N}, \mathrm{ChInN})$ receives the number $\mathrm{N}$ and returns in ChInN the list of the ASCII codes of the characters comprising the representation of $\mathrm{N}$ as a decimal number. Then, it tuses the well-known list concatenation procedure app/3. Note that lists are represented in this example by using quoted strings. The call atom_codes (File,Fname) receives in Fname 7. list of ASCII codes and returns in File the atom (constant) made up of the corresponding characters. Also, a call such as open (File, Mode, F) opens the file named File and returns in $F$ the strean associated to the file. The argument Mode can have any of the values: read. write, or append. Procedures number_codes $/ 2$, atom_codes $/ 2$, and open $/ 3$ are ISO-standard Prolog proce- 
dures, and thus they are available in CiaoPP (in the iso-prolog library).

In the following, we assume that all rule heads are normalized, i.e., $H$ is of the form $p\left(X_{1}, \ldots, X_{n}\right)$ where $X_{1}, \ldots, X_{n}$ are distinct free variables. This is not restrictive since prograns can always be nomalized, and it will facilitate the presentation of the algorithms later. For instance, the procedure createstreams of Example 2.1 in normalized form is as follows.

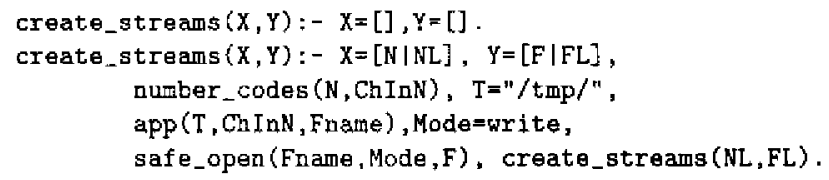

\subsection{Abstract Interpretation}

In Abstract Interpretation, ${ }^{16 !}$ programs are interpreted over an abstract domain $\left(D_{\alpha}\right)$ which is simpler than the corresponding concrete domain $(D)$. An abstract value is a finite representation of a possibly infinite set of actual values in the concrete domain. Otr approach relies on the abstract interpretation theory. ${ }^{13)}$ where the set of all possible abstract semantic values which represents $D_{\alpha}$ is usually a complete lattice or cpo which is ascending chain finite. However, for this study, abstract interpretation is restricted to complete lattices over sets. both for the concrete $\left(2^{D}, \subseteq\right)$ and abstract $\left\langle D_{\alpha x} . \sqsubseteq\right\rangle$ domains. Abstract values and sets of concrete values are related via a pair of monotonic mappings $\langle\alpha, \gamma\rangle$ : abstraction $\alpha: 2^{D} \rightarrow D_{\alpha}$, and concretization $\gamma: D_{\alpha} \rightarrow 2^{D}$, such that $\forall x \in 2^{D}: \gamma(\alpha(x)) \supseteq x$ and $\forall y \in D_{\alpha}: \alpha(\gamma(y))=y$. In general $\sqsubseteq$ is induced by $\subseteq$ and $a$. Similarly, the operations of least upper bound ( $\sqcup$ ) and greatest lower bound $(\Pi)$ mimic those of $2^{D}$ in a precise sense.

In our framework, the safcty properties that the execution of a program must satisfy are defined as abstract substitutions. This allows us to express properties in terms of abstract domains. In turn, different domains can be used for different safety properties. The abstract (or description) domain we use in our examples is the following regular type domain. ${ }^{\text {t? }}$

\section{Example 2.2 (regular type domain)}

We refer to the regular type domain as eterms, since it is the name it has in CiaoPP. Abstract substitutions in eterns, ${ }^{44}$ over a set of variables $V$, assign a regular type to each variable in $V$. We use in our examples term as the most general type (i.e., term $\equiv T$ corresponds to all possible terms). We also allow parametric types such as 1 ist $(\mathrm{T})$ which denotes lists whose elements are all of type T. Type list is equivalent to list (term). Also, list(T) $\sqsubseteq$ list $\sqsubseteq$ term for any type $T$. The least general substitution $\perp$ assigns the empty set of values to each variable and indicates that the corresponding program point is unreachable.

Apart from predefined types. in the eterms domain, the user can define 
regular types by means of Regular Unary Logic programs. ${ }^{21+1}$ For instance, in the context of mobile code, it is a safety issue whether the code tries to access files whicl are not related to the application in the machine consuming the code. A very simple safety policy can be to enforce that the mobile code only accesses temporary files. In a UNIX system this can be controlled (under some assumptions) by ensuring that the file resides in the directory $/ \mathrm{tmp} /$. The following regular type safe name defines this notion of safety, where the regtype declarations are used in Ciaopp to define new regular types:

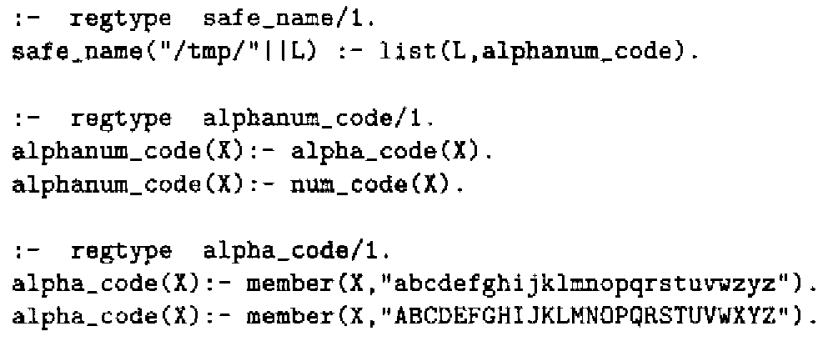

The regular type num_code $(X):-$ member $(X, " 0123456789 . e E+-")$ is predetincd in the system. The abstract property made up of substitution $\{\mathrm{X} \mapsto$ safe name $\}$ expresses that $X$ is bound to a string which starts with " $/ \mathrm{tmp} /$ " followed by a "ist of alpha-numerical characters (we use "I " to denote list concatenation). In the following, we write simply safe name $(X)$ to represent it. The crucial point. here is llat safe names cannot contain (back-)slashes. As a result, there is no way safe_names caul access files outside the /tmp/ directory.

\section{A General View of Abstraction-Carrying Code}

An abstract interpretation-based certifier is a function certifier : Prog $\times$ $A$ Dom $\times$ AInt $\rightarrow$ ACert which for a given program $P \in$ Prog, an alsstract domain $D_{\alpha} \in A D o m$ and a safety policy $I_{c r} \in A$ Int generates a certificate Cert $t_{c r} \in$ $A C e r t$, by using an abstract interpreter for $D_{\alpha}$. which entails that $P$ satisfies $I_{\alpha}$. This abstract safety specification $I_{\alpha}$ embodies the safety requirements, i.e., it is an expression of the consumer's safety expectations. Its formalization in our context is the main issue of Section 4 . In the following. we denote that $I_{\alpha}$ and $C$ ert $t_{r}$ are specifications given as abstract semantic values of $D_{\alpha}$ by using the same $\alpha$. The basics for defining such certifiers (and their corresponding checkers) in $\mathrm{ACC}$ are summarized in the following five points and Equations:

Analysis. We consider the class of fixed-point semantics in which a (monotonic) semantic operator, $S_{P}$, is associated to each program $P$. The meaning of the program. $\llbracket P \rrbracket$, is defined as the least fixed point of the $S_{P}$ operator. i.e., $\llbracket P \rrbracket=$ Ifp $\left(S_{P}\right)$. If $S_{P}$ is continuous, the least fixed point is the limit of an iterative process involving at most $\omega$ applications of $S_{P}$ starting from the bottom element.

* Adationally such types are inferred by the system iudepenclently of the pre- ar userdefined types. 
of the lattice. Consider the operator $S_{p}^{* x}$ which is the abstract counterpart of $S_{P}$. Using abstract interpretation, we can usually only compute $\llbracket P \rrbracket_{\alpha}$, as $\llbracket P \rrbracket_{\alpha}=$ $\operatorname{lfp}\left(S_{P}^{\alpha}\right)$.

$$
\operatorname{anaIyze}\left(P . D_{\alpha}\right)=\operatorname{lf} p\left(S_{P}^{\alpha}\right)=\prod P \prod_{\alpha}
$$

Correctness of analysis ensures that $\llbracket P \rrbracket_{\alpha}$ safely approximates $\llbracket P \rrbracket$, i.e. $\llbracket P \rrbracket \in$ $\gamma\left(\llbracket P \rrbracket_{\alpha}\right)$.

The generation of Equation (1) is the main issue of Section 5.

Verification Condition. Let Cert $_{\alpha}$ be a safe approximation of $P$. If all abstract safety specification $I_{\alpha}$ can be proved w.r.t. Cert $_{\alpha}$, then $P$ satisfies the safety policy and $\operatorname{Cert}_{\alpha}$ is a valid certificate:

$$
\text { Cert }{ }_{\alpha} \text { is a valid certificate for } P \text { w.r.t. } I_{\alpha} \text { if Cert } \_\sqsubset I_{\alpha}
$$

Equation (2) is explained in detail in Section 6.

Certifier. Together, equations (1) and (2) define a certitier which provides program fixpoints, $\llbracket P \rrbracket_{\alpha}$, as certificates which entail a given safety policy, i.e., by taking Cert C $_{\alpha}=\|P\|_{\alpha}$. Note that the two equations define the function certifier specified above.

Checking. A checker is a function checker : Prog $\times A$ Dom $\times A C e r t \rightarrow$ bool which for a program $P \in$ Prog, an alsstract domain $D_{\alpha} \in A D o m$ and a certificate Cert $_{\alpha \mathrm{c}} \in A$ Cert checks whether Cert $_{\alpha}$ is a fixpoint of $S_{p}^{\alpha}$ or not:

$$
\operatorname{check}\left(P, D_{\alpha}, \text { Cert } t_{\alpha}\right) \text { returns truf iff }\left(S_{P}^{\alpha}\left(\operatorname{Cert}_{\alpha x}\right) \equiv \operatorname{Cert}_{\alpha}\right)(3)
$$

The definition of a check function satisfying Equation (3) is the purpose of Section 7 .

Verification Condition Regeneration. To retain the safety guarantees, the consumer must regenerate a trustworthy verification condition -Equation 2- and use the incoming certificate to test for adherence of the safety policy.

$$
P \text { is trusted iff Cert } \alpha \sqsubseteq I_{\alpha}
$$

A fundamental idea in ACC is that, while analysis -equation (1)- is an iterative process which may traverse (parts of) the abstraction more than once until the fixpoint is reached, checking -equation (3)- is guaranteed to be done in a single pass over the abstraction (a single application of $S_{P}^{\alpha}$ ).

\section{\$4 An Assertion Language to Specify the Safety Policy}

The aim of this section is to present the safety policy $I_{\alpha} \in$ AInt within the ACC approach. The purpose of a safety policy is to specify precisely the (abstract) conditions under which the execution of a program is considered safe. Assertions are syntactic objects which allow expressing a wide variety of highlevel properties of (in our case CLP-) programs. Examples are assertions which state information on entry points to a program module, assertions which describe properties of predefined procedures (built-ins), assertions which provide some 
type declarations, cost bounds, etc. They will allow us to have an expressive class of safety policies in the context of (constraint) logic programs. Int uitively, we assume that a program will be accepted at the receiving end, provided all properties stated within assertions can be checked, i.e., the abstract specification $I_{\Omega}$ expressed in the assertions detcrmines the safety policy. This can be a policy agreed a priori or exchanged dynamically.

The original assertion language ${ }^{37}$ available in CiaoPP is composed of several assertion schemes. Among them, we only consider the following two schemes in AIrt for the purpose of this article, which intuitively correspond to traditional pre- and postconditions on procedures:

calls $\left(B,\left\{\lambda_{\text {Pre }}^{1} ; \ldots ; \lambda_{\text {Pre }}^{n}\right\}\right)$ : This assertion scheme is used to express properties which should hold in any call to a given procedure, in a sinuilar way to the traditional precondition. $B$ is a procedure descriptor, i.e.. it has a procedure name (predicate symbol) as main functor and all argunents are distinct free variables, and $\lambda_{p_{r e}}^{i}, i=1, \ldots, n$, are abstract properties of execution states. The resulting assertion should be interpreted as "in all activations of $B$ at least one property $\lambda_{P r e}^{i}$ should hold in the calling state."

success $\left(B,\left[\lambda_{\text {Pre }},\right] \lambda_{\text {Post }}\right)$ : This assertion scheme is used to describe a postcondition which must hold on each success state for a given procedure. $B$ is a procedure descriptor, and $\lambda_{p \text { re }}$ and $\lambda_{P o s}$ are abstract properties about execution states. $\lambda_{P r e}$ is optional and must be evaluated w.r.t. the description at the calling state to the procedure while condition $\lambda_{\text {Past }}$ is evaluated at the success state. If the optional $\lambda_{\text {Pre }}$ is present, then $\lambda_{\text {post }}$ is only required to hold in those success states which correspond to call states satisfying $\lambda_{P \text { re. }}$. Note that several success assertions for the same procedure aud with different $\lambda_{\text {fre }}$ may be given.

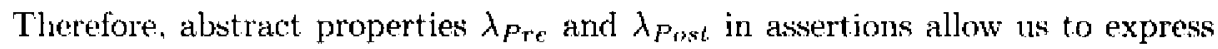
$I_{\alpha} \in A I n t$ as conditions. in terms of an abstract domain $D_{\alpha}$, that the execution of a program must satisfy. Each condition is an abstract substitution corresponding to the variables in some literal.

In existing approaches, safety policies usually correspond to some variants of type safety (which may also control the correct access of memory or array bounds ${ }^{351}$ ). In our system, the co-existence of several domains allows expressing a wide range of properties using the assertion language. They include several classes of safety policies based on modes, types, non-failure, termination, determinacy, non-suspension, non-floundering, cost bounds, and their combinations.

In genemal. it is the task of the compiler designer to define the safety policies associated with the predefined systcm procedures. In addition to these issertions, the user can optionally provide further assertions manually for userdefined procedures. As depicted in Fig. 1, given an initial program $P$, we first define its Safety Policy $I_{\alpha}$ as a set of assertions $A S$ in the context of an abstract domain $D_{\alpha}$. The domain is appropriately chosen among a repertoire of abstract 


\begin{tabular}{|c|}
\hline $\begin{array}{c}\text { calls (number_codes }(X, Y),\{(\operatorname{mum}(X) ; 1 \text { ist }(Y, \text { num_code }))\}) \\
\text { calls(aton_codes }(X, Y),\{(\operatorname{constant}(X) ; \operatorname{string}(Y))\}) \\
\text { calls(open }(X, Y, Z),\{\text { constant }(X), \text { iomode }(Y)\})\end{array}$ \\
\hline calls (safe,open (Frame,-,.), \{safe.name(Frame) $\})$ \\
\hline $\begin{array}{l}\text { success (number_codes }(X, Y), T,\{\operatorname{num}(X), \operatorname{list}(Y, \text { num_code })\}) \\
\text { success }\left(\operatorname{atom} \_\operatorname{codes}(X, Y), T,\{\operatorname{constant}(X), \operatorname{string}(Y)\}\right) \\
\text { success }(\operatorname{open}(X, Y, Z), T,\{\operatorname{constant}(X), \text { iomode }(Y), \operatorname{stream}(Z)\})\end{array}$ \\
\hline
\end{tabular}

Fig. 2 Assertions for the Example

domans available in the system. The assertions are obtained from the assertions for system procedures and those provided by the user. Let us illustrate this process by means of an example.

\section{Example 4.1 (Safety Policy)}

Figure 2 shows the assertions which aro relevant to the program in our running exauple. 'The first four rows correspond to calls assertions, whereas the last three are success assertions. Out of the four calls, the first three are predefined in the system. For example, the first one states that calls to number_codes $/ 2$ have to be performed with the first argument bound to a number or the second argument bonnd to a list of num_code, which is a predefined type that includes the ASCII characters required for representing floating point (and integer) mumbers as strings. The last calls assertion is for procedure safe_open and provides a simple way to guarantee that all subsequent calls to open ale safe. It can be read as "the calling conventions for procedure safe_open require that the first argument be a safe name." The success assertion for open is predefined in our system and requires, upon success, the first variable to be of type constant, the second a proper io mode and the last one of type stream.

In contrast to traditional approaches. assertions are not compulsory for every procedure. Thus, the user can decide how much effort to put into writing assertions: with more of them, the intended semantics (and thus the partial correctness) of the program is described nore cxhaustively and more possibilities arise for detecting problems. Indeed, pre- and post-conditions are frequently provided by programmers, specially in the case of libraries, since they are often easy to write and very useful not only for verification but also for example for generating program documentation. Nevertheless, the analysis algorithm is able to obtain safe approximations of the program behavior even if no assertions are given. This is not always the case in other approaches such as classical program verification, in which loop invariants are actually required. Such invariants are hard to find and existing automated techniques are not always sufficient to infer them, so that often they have to be provided by liand.

Note that the three success assertions shown in the example correspond to library procedures. Such assertions can be verified beforehand, and indeed they are verified in our implemontation assiming that calls to such procedures satisfy the corresponding calls assertions. Unfortunately, calls assertions for library procedures cannot be verified beforehand, since programs which use such procedures may do so incorrectly. Thus, in order to guarantee that the safety policy holds we only need to prove that the four calls assertions in Fig. 2 hold. 


\section{$\$ 5$ Generation of Program Abstractions}

This section introduces part of the certification process, represented to the left of Fig. 1. carried ont by the producer, uamely the generation of an abstraction $\llbracket P \rrbracket_{r k}$ which safely approximates the behaviour of the program (Equation 1 in Section 3). The generation of the verification condition from this certificate (Equation 2) will be discussed in the next section.

\subsection{The Analysis Graph}

Global program analysis is becoming a practical tool in constraint logic program compilation in which information about calls, answers, and the effect of the constraint store on variables at different program points is computed statically. ${ }^{10,21,33,41,4: 3)}$ Essentially, an analyzer returns an abstraction of $P^{\prime}$ 's execution in terms of the abstract domain $D_{n}$. The underlying theory, formalized in terms of abstract interpretation ${ }^{167}$ and the related implementation technicues are well understood for several general types of analysis and. in particular, for top-down analysis of Prolog. ${ }^{8.14 .18 .19 .28 .33)}$ Several generic analysis engines. such as the one implemented in the CiaoPP system. ${ }^{221}$ PLAl, ${ }^{31,331}$ GAIA, ${ }^{1+1}$ and the $\operatorname{CLP}(\mathcal{R})$ analyzer, ${ }^{251}$ facilitate the construction of such top-down analyzers. As shown in Fig. 1 in principle the analyzer is domain-independent. This allows plugging in different alst ract domeins provided suitable interfacing functions are defined. From the user's point of view, it is sufficient to specify the particular abstract domain desired during the generation of the safety assertions. Different donains yield analyzers which provide different kinds of information. degrees of accuracy, and efficiency. The core of each generic abstract interpretation-based engine is an algorithm for efficient fixed-point computation. ${ }^{13.31 .33 .3 \%}$

In order to analyze a program, traditional (goal dependent) abstract interpreters for (C)LP programs, such as those referenced above, receive as input, in addition to the program and the abstract domain. a set $S$ of calling patterns. Such calling patterns are pairs of the forn $A: C P$ where $A$ is a procedure descriptor and $C P$ is an abstract substitution (i.e.. a condition of the run-time bindings) of $A$ expressed as $C P \in D_{\alpha}{ }^{*}$ Given a program $P$ and a set $S$ of calling patterns in the context of an abstract domain $D_{\alpha}$. the analyzer constructs an and-or graph (or analysis graph) for $S$ which can be viewed as an abstraction $\llbracket P \rrbracket_{r x}$, i.e., a finite representation of the (possibly infinite) set of (possibly infinite) AND-OR trees explored by the concrete execution of initial calls described by $S$ in $P .{ }^{\left.{ }^{3}\right)}$ Finiteness of the program analysis graph (and thus termination of analysis) is achieved by considering abstract domains with certain characteristics (such as being finite, or of finite height, or without infinite assending chains) or by the use of a widening operator. ${ }^{16)}$

$\because$ In principle. calling patterns are only required for exported procedures. The atralysis algorithm is able to generate them automatically for the remaining internal procedures. Nevertheless, they can still be automatically genetated by assuming $T$ (i.e., no initial lata) for all exported procedures (although the idea is to improve this information in the initial calling patterns). 


\section{Example 5.1}

Consider our running example and assume that we are interested in analyzing it for the call create_streams(X,Y) with initial description list $(X$, num $)$, indicating that we wish to analyze it for any call to create_streams $/ 2$ with the first argument being a list of numbers. In essence the analyzer must produce the program analysis graph given in Fig. 3.

The graph has two sorts of nodes. Those which correspond to literals are called "OR-nodes". The OR-node in the root:

$$
{ }^{\text {list }(X, n u m)} \text { create_streams }(X, Y)^{\text {inst }(X, \pi u m) \text {, list }(Y, \text { stream })}
$$

indicates that when the literal create streams $(X, Y)$ is called with description $\operatorname{list}(X$, numb) the answer (or success) substitution computed is $\{$ list $(X$, num $)$. $\operatorname{list}(Y$, stream $)\}$

Those nodes which correspond to rules are called "AND-nodes." In Fig. 3, they appear within a dashed box and contain the head of the corresponding clause. Each AND-node has as children as many OR-nodes as literals there are in the body. We indicate with the symbol $\square$ that the rule is a fact with no literals in the body. And the symbol o denotes that the code for this procodure is not available (i.e., it is an external procedure). These rules are annotated by abstract descriptions (referenced by numbers $0, \ldots, 13$ whose corresponding description appears to the bottom of the figure) at each program point when the rule is executed from the calling pattern of the node connected to the rules. The program points are at the entry to the rule, the point between each two

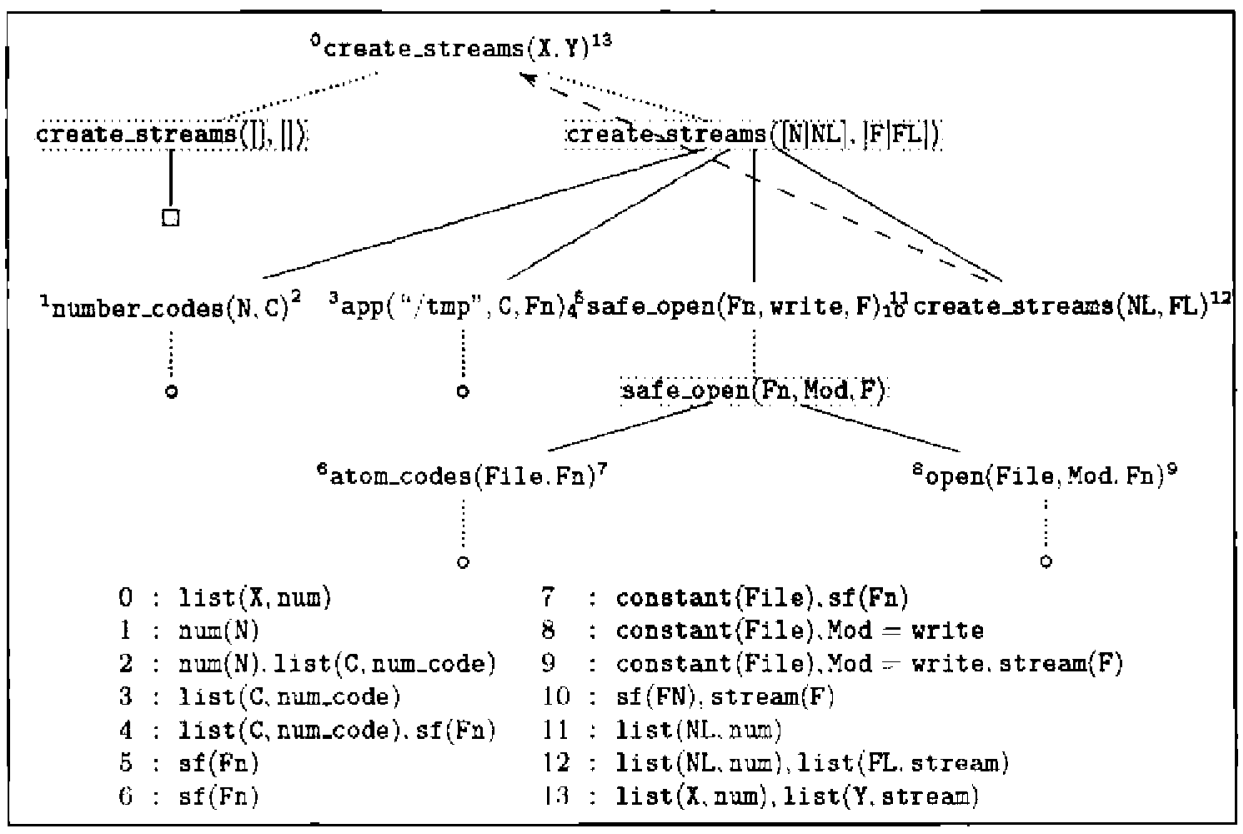

Fig. 3 Analysis Graph 
literals, and at the return from the call. If a child OR-mode is already in the tree, it is not further expanded and the currently available answer is used. For instance, the analysis graph in Fig. 3 contains two occurrences of the abstract literal create_streams $(X, Y): \operatorname{list}(X, n u m)$ (modulo renaning), but only one of them has been expanded. This is depicted by an arrow from the non-expanded occurrence of create_streams $(X, Y): \operatorname{list}(X, n u m)$ to the expanded one. How this program analysis graph is constructed and the meaning of the duxiliary type sf is detailed in Example 5.2 below.

For a given program and set of calling patterns there may be many different analysis graphs. However, for a given set of initial calling patterns, progran, and abstract operations on the abstract domain, there is a unique least analysis graph which gives the most precise information possible.

\subsection{The Analysis Algorithm}

The aim of this section is two-fold. First, we introtuce an analysis algorithm which computes an analysis graph. Second, we identify the fragnent of the information stored in such graph which is sufficient in order to play the role of safety certificate, written as $C$ ert $t_{c r}=\llbracket P \rrbracket_{\Omega}$.

The analysis algorithm presented is an extension of the generic analysis algorithm $\mathrm{in}^{22)}$ in order to handle (constraint) logic programs with external (including imported) procedures. For this, our analyzers rely again on assertionbased techniques. ${ }^{3 i}$ Intuitively, our analyzer trusts the information stated in the success assertions for external procedures by considering the answer pattern in them a safe approximation of the concrete answer pattesns. In our algoritlun. the arlalysis of external procedures is handled by the function Atrust which is introduced below. The details about analysis of modular programs can be found in $^{363}$ and are out of the scope of this article.

The program analysis graph is implicitly represented in the algorithm by means of two data structures, the answer table and the dependency arc table. Given the information in these. it is straight forward to construct the grapl and the associated progran point annotations. The answer table contains entries of the form $A: C P \mapsto A P$, where $A$ is always a base form and $A P$ and $C P$ are abstract substitutions. This corresponds to OR-nodes in the analysis graph of the form ${ }^{C P} A^{A P}$. A dependency arc is of the form $H_{k}: C P_{0} \Rightarrow\left[C P_{1}\right] B_{k, i}$ : $C P_{2}$. This is interpreted as follows: if the rule with $H_{k}$ as head is called with. description $C P_{0}$ then this canses literal $B_{k, i}$ to be called with description $C P_{2}$. The remaining part $C P_{1}$ is the program amotation just before $B_{k, i}$ is reached and contains information about all variables in rule $k$. Note that the annotation $C P_{1}$ is not really necessary, as it could be recomputed, but it is included for efficient recomputation. As we will see below, dependency ares are used for enforcing recomputation until a fixed point is reached.

Int uitively, the antasis algorithm is a graph traversal algorithm which places entries in the answer table and dependency arc table as new nodes and ares in the program analysis graph are encountered. In order to guide computation. we need at third data structure for storing the events which are to be handled. 
Events are of three forms:

newcall $(A: C P)$ which indicates that a new calling pattern for literal $A$ with description $C P$ has been encountered.

$\operatorname{arc}\left(H_{k}: C P_{0} \Rightarrow\left[C P_{1}\right\rfloor B_{k, i}: C P_{2}\right)$ which indicates that the body of rule $k$ needs to be processed from position $i$, i.e., from body literal $B_{k, i}$ until the end of rule $k$, using the annotation $C P_{1}$.

- updated(A : CP) which indicates that the answer description to calling pattern $A$ with description $C P$ has been changed.

Rather than simply 11sing a stack or a quene for storing events, which would result in a depth-first or a breadth-first traversal, respectively, we use a prioritized event queue. This allows capturing the sophisticated graph traversal strategies used in optimized fixed-point algorithms. For example, note that there are three different classes of events and we may be interested in assigning different priorities to each class of events. Coming up with an efficient strategy is out of the scope of this paper. A detailed discussion with concrete proposals can be found. ${ }^{3 m}$

Our analysis algorithm is given in Fig. 4. It is defined in terms of five abstract operations on the abstract domain $D_{\alpha}$ of interest:

- Arestrict $(C P, V)$ performs the abstract restriction of a description $C P$ to the set of variables in the set $V$, denoted vars $(V)$;

- Aextend $(C P, V)$ extends the description $C P$ to the variables in the set $V$;

- Atrust $(A, C P)$ returns an answer pattern for an external procedure $A$ which is a safe approximation of the concrete answer patterns for all call patterns described by $C P$;

Aconj $\left(C P_{1}, C P_{2}\right)$ performs the abstract conjunction of two descriptions;

Alub $\left(C P_{1}, C P_{2}\right)$ performs the abstract disjunction of two descriptions.

Apart from the parametric description domain-dependent functions, the algorithm has several other undefined functions. The functions add_event and next_event respectively add an event to the priority quene and return (and delete) the event of highest priority.

When ar event being added to the priority queue is already in the priority queue, a single event with the maxinum of the priorities is kept in the queue. When an arc $H_{k}: C P \Rightarrow\left[C P^{\prime \prime}\right] B_{k, i}: C P^{\prime}$ is added to the dependency arc table, it replaces any other arc of the form $H_{k}: C P \Rightarrow[-] B_{k, i}:$ - in the table and the priority quene. Similarly when an entry $H_{k}: C P \mapsto A P$ is added to the answer table. it replaces any entry of the form $H_{k}: C P \mapsto$. Note that the underscore (-) matches any description, and that there is at most one matching entry in the dependency arc table or answer table at any time. The function initial_guess returns an initial guess for the answer to a new calling pattern. The default value is $\perp$ but if the calling pattern is more general than an already computed call then its current value may be returned.

The algorithm centers around the processing of events on the priority quele in main_loop, which repeatedly removes the highest priority event and calls the appropriate event-handling function. When all events are processed it calls remove_useless_calls. This procedure traverses the dependency grapli given 


\begin{tabular}{|c|c|}
\hline 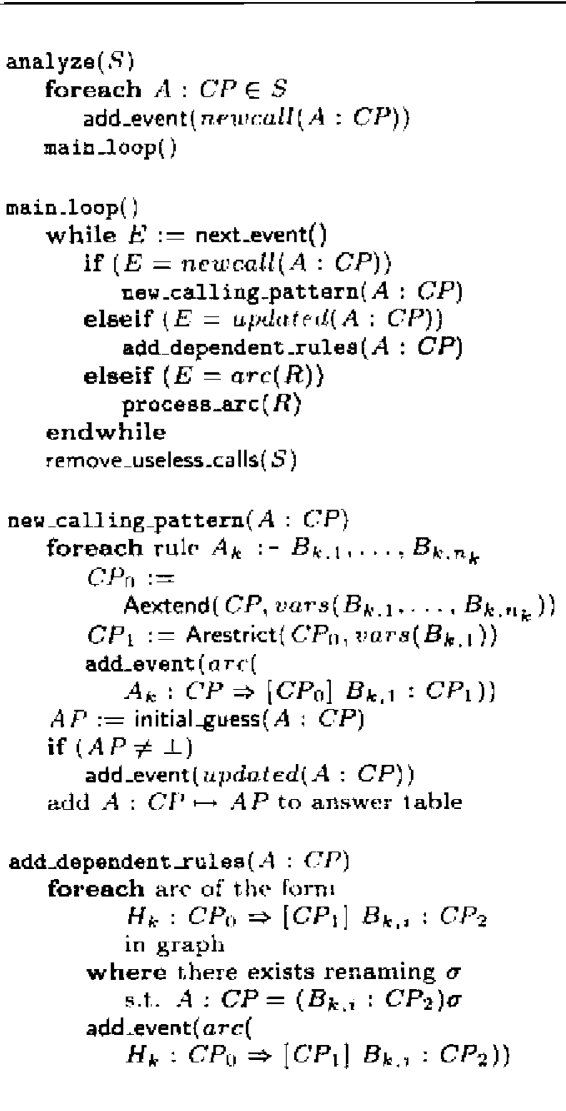 & 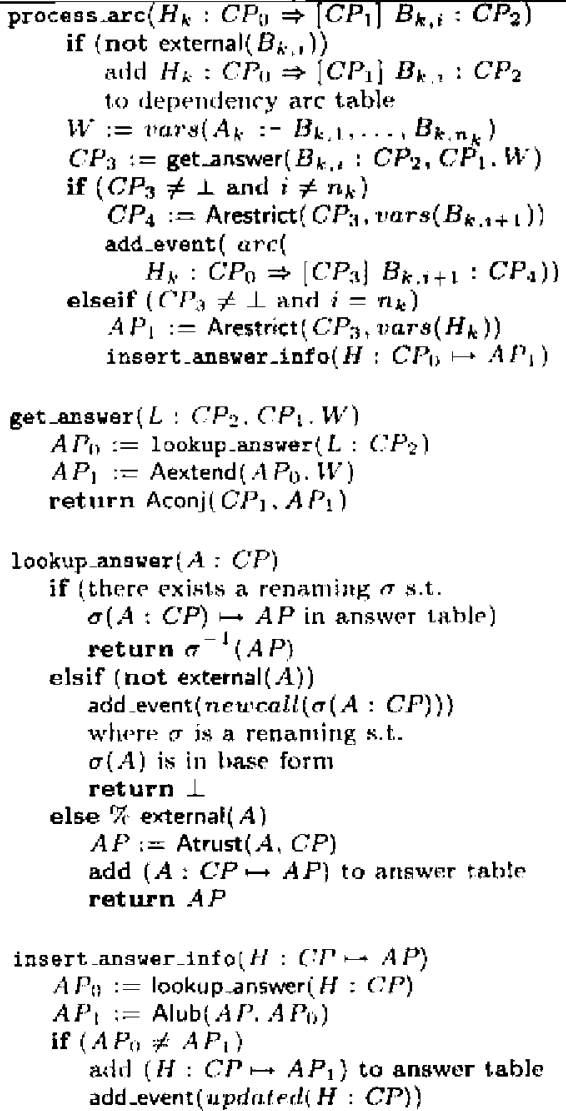 \\
\hline
\end{tabular}

Fig. 4 Fixed-point Analyzer

by the dependency ares from the initial calling patterns $S$ and nuarks those entries in the dependency arc and answer table which are reachable. Those remaining are removed.

The function new_calling-pattern initiates processing of the rules in the definition of the intermal literal $A$, by adding are events for each of the first literals of these rnles, and determines an initial answer for the calling pattern and places this in the talsle. The function add_dependent_rules adds arc cvertis for each dependency arc which depends on the (alling jattern $A$ : $C P$ for which the answer has been updated. The function process_arc performs the core of the analysis. It performs a single step of the left-to-right traversal of a rule body. If the literal $B_{k, 2}$ is not for an external procedure, the arc is added to the dependency arc table. 'The current answer for the call $B_{k, i}: C P_{2}$ is conjoined with the description $C P_{1}$ from the program point innediately before $B_{k, i}$ to obtain the description for the program point rffer $B_{k, i}$. This is either now to generate a new arc event to process the next litcral in the rule if $B_{k, i}$ is not the last literal: otherwise the new answer for the rule is combined with the 
current answer in insert_answer_info. The function get_answer processes a literal. The current answer to that literal for the current description is looked up; then this answer is extended to the variables in the rule the literal ocmirs in and conjoined with the current description. The function lookup_answer first looks up an answer for the given calling pattern in the answer table and if it is not found and the procedure is local, it places a newcall event. Otherwise (the procedure is extermal), it uses Atrust to obtain a safe answer pattern. Finally, insert_answer_info, updates the answer table entry when a new answer is found.

\section{Theorem 5.1 (correctness ${ }^{21}$ )}

For a program $P$ and calling patterns $S$, the analysis algorithm of Fig, 4 returns an answer table and dependency arc table which represents the least program analysis graph of $P$ and $S$.

A central idea in ACC is that, for certifying program safety, it suffices to send the information stored in the analysis answer table. The theory of abstract. interpretation guarantees that the answer table is a safe approximation of the runtime behavior ( see $^{8.22 .39)}$ for details). In contrast to this analysis algorithm, a simple checker can be designed for validating the answer table without requiring the use of the arc dependency table at all (as we show in Sect. 7).

\subsection{An Example}

The following example illustrates the operation of the fixed-point algorithm. It. shows how the create streams program is analyzed, to obtain the program analysis graph shown in Fig. 3. We use in our example well-known abstract operations for a regular type domain, in particular, the operations formatized in ${ }^{44)}$ for the eterms donain described in Example 2.2. For the analysis of library procedures. we assitne that the parametric routine Atrust returns the abstract descriptions which appear in the three success assertions shown in Fig. 2 for the corresponding library procedures. This can be done safely because, as already mentioned, such success assortions have been verified beforehand.

\section{Example 5.2}

Analysis begins from an initial set $S$ of calling patterns. In our example $S$ contains the single calling pattern createsstreams $(X, Y): \operatorname{list}(X, n u m)$. For brevity, variables which do not appear in abstract substitutions are assumed to be "term". Also $\operatorname{nil}(X)$ indicates that $X$ is the mpty list. The first step in the algorithm is to add the initial calling patterns as a newcall event to the priority queue. After this the priority queue contains

$$
\text { neucall(create streams }(\mathrm{X}, \mathrm{Y}): \operatorname{list}(X, n \text {, } u m) \text { ) }
$$

and the answer and dependency arc tables are empty. The newcall event is taken from the event queue and processed as follows: for each rule defining create streams, an arc is added to the priority queue which indicates the rule body which nulst be processed from the initial literal. An entry for the new calling pattern is added to the answer table with an initial guess of $\perp$ as the answer. The data structures are now: 
priority queue:

$\operatorname{arc}(\operatorname{create}$ streans $(X, Y): \operatorname{list}(X, n u m) \Rightarrow[\operatorname{list}(X, n u m)] X=[]: \operatorname{list}(X, n u m))$

$\operatorname{arc}\left(\mathrm{create} \_\operatorname{streams}(\mathrm{X}, \mathrm{Y}): \operatorname{list}(X, \operatorname{num}) \Rightarrow[\operatorname{list}(X, \operatorname{num})] \mathrm{X}=[\mathrm{N} \mid \mathrm{NL}]: \operatorname{list}(X\right.$, num $\left.)\right)$

ariswer table:

create_streams $(X, Y):$ list $(X, n u m) \mapsto \perp$

dependency arc table:

no entries

An arc on the event queue is now selected for processing, say the first. The routine get_answer is called to find the answer pattern to the literal $X=[]$ with description list $(X, m u m)$. As the literal is an external constraint, the parametric routine Atrust is used. It returns the answer pattern $\{$ list $(X . n u m), \operatorname{mil}(X)\}$. A new arc is added to the priority queue which indicates that the second literal in the rule body must be processed. The priority queue is now

$$
\begin{aligned}
& \operatorname{arc}\left(\operatorname{create} \_\operatorname{streams}(X, Y): \operatorname{list}(X, n u m) \Rightarrow[\operatorname{list}(X, n u m), \operatorname{mil}(X)] \mathrm{Y}=[]:\{\}\right) \\
& \operatorname{arc}(\operatorname{create} \operatorname{streams}(\mathrm{X}, \mathrm{Y}): \operatorname{list}(X, n u m) \Rightarrow[\operatorname{list}(X, n u m)] \mathrm{X}=[\mathrm{N} \mid \mathrm{NL}]: \operatorname{list}(X, n u m))
\end{aligned}
$$

The answer and dependency arc table remain the same.

Again, an arc on the event queue is selected for processing. say the first. As before, get_answer and Atrust are called to get the next annotation flist $(X$, num $)$, nil $(X), n i l(Y)\}$. This time, as there are no more literals in the body, the entry for create_streams $(X, Y): \operatorname{list}(X, n u m)$ in the answer table is updated. Alub is used to find the least upper bound of the now answer $\{l i s t(X, n u m), \operatorname{nil}(X), \operatorname{mil}(Y)\}$ with the old answer $\perp$. This gives $\{l i s t(X, n u m), n i l(X)$, nil(Y)\}. The entry in the answer table is updated, and an updated event is placed on the priority queue. The data structures are now:

priority queur:

uploted(create.streams $(\mathrm{X}, \mathrm{Y}): \operatorname{list}(X, n u m))$

arc(create_streams $(\mathrm{X}, \mathrm{Y}): \operatorname{list}(X, n u m) \Rightarrow$

$$
[\operatorname{list}(X, n u m)] \mathrm{X}=[\mathrm{N} \mid \mathrm{NL}]: \operatorname{list}(X . n u m))
$$

answer table:

create.streams $(\mathrm{X}, \mathrm{Y}): \operatorname{list}(\mathrm{X}, \mathrm{num}) \mapsto\{\operatorname{list}(\mathrm{X} . \operatorname{num})$ mil(X),nil(Y)\}

dependency arc table:

no entries

The updated event can now be processed. As there are no entries in the dependency arc table, nothing in the current program analysis graph depends on the answer to this call, so nothing needs to be recomputed. The priority queue now contains

$$
\begin{aligned}
& \operatorname{arr}(\operatorname{create} \operatorname{streams}(\mathrm{X}, \mathrm{Y}): \operatorname{list}(X . \operatorname{mum}) \Rightarrow \\
& [\operatorname{list}(X, m m m)] \mathrm{X}=[\mathrm{N} \mid \mathrm{NL}]: \operatorname{list}(X . n u m))
\end{aligned}
$$

The answer and dependency arc table romain the sane. Similarly to before we process the arc. giving rise to the new priority queue

$\operatorname{arc}($ create streams $(\mathrm{X}, \mathrm{Y}): \operatorname{list}(\mathrm{X} . \mathrm{num}) \Rightarrow$ 


$$
[\operatorname{list}(X, \operatorname{mum}), \operatorname{mum}(N), \operatorname{list}(N L, \operatorname{mum})] \mathrm{Y}=[F \mid F L]:\{\}) .
$$

The arc is processed to give the priority queue

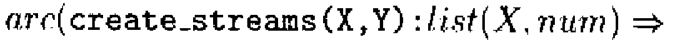

$$
\begin{aligned}
& {[\operatorname{list}(X, n u m) . \operatorname{lum}(N), \operatorname{list}(N L, n u m), Y=[F \mid F L]]} \\
& \text { number.codes }(\mathrm{N}, \mathrm{ChInN}): \operatorname{num}(N) \text { ) }
\end{aligned}
$$

Note that CiaoPP creates the regular type rt2 to represent a term whose toplevel functor is a list constructed with $F$ as head and FL as tail. For simplicity, we just write this description as $Y=[F \mid F L]$ in the following.

This time, because number_codes $(\mathrm{N}, \mathrm{ChInN})$ is an external literal, the parametric routine Atrust is used and no dependency is stored (as success patterms for external procedures are never updated). As a result, the data structures are now:

priority queue:

$\operatorname{arc}(\operatorname{create} \operatorname{streams}(\mathrm{X}, \mathrm{Y}): \operatorname{list}(X, \operatorname{mum}) \Rightarrow[\operatorname{list}(X, n u m), \operatorname{num}(N)$, list $(N L$, num $), Y=[F \mid F L]$.list(ChInN,num_code $)] \mathrm{T}=" / \mathrm{tmp} / ":\{\})$ answer table:

create_streams $(X, Y): \operatorname{list}(X, n u m) \mapsto \operatorname{list}(X, n u m), \operatorname{nil}(X), \operatorname{nil}(Y)$

number_codes (N, ChInN) : num( $N) \mapsto \operatorname{num}(N)$, list (ChInN, num_code) dependency arc table:

no entries

Following the analysis. we process the unique arc in the priority queue, obtaining the new priority queue:

priority queue:

$\operatorname{arc}($ create_streams $(X, Y): \operatorname{list}(X, n u m) \Rightarrow[\operatorname{list}(X, \operatorname{num}), \operatorname{num}(N)$, list $(N L, n u m), Y=[F \mid F L], \operatorname{list}(C h I n N$, num_code $), T=" /$ tmp $/ "]$ $\operatorname{app}(\mathrm{T}, \operatorname{ChInN}$, Fname $): T=" / \mid m p / ", \operatorname{list}\left(\right.$ ChInN,$\left.\left.n u m m_{-} \operatorname{code}\right)\right)$

Similarly as done so far. and skipping the intermediate steps, we obtain finally the following data structures in which, the dependency arc table contains a different arc for each one of the literals in the second rule of create streams which are not external.

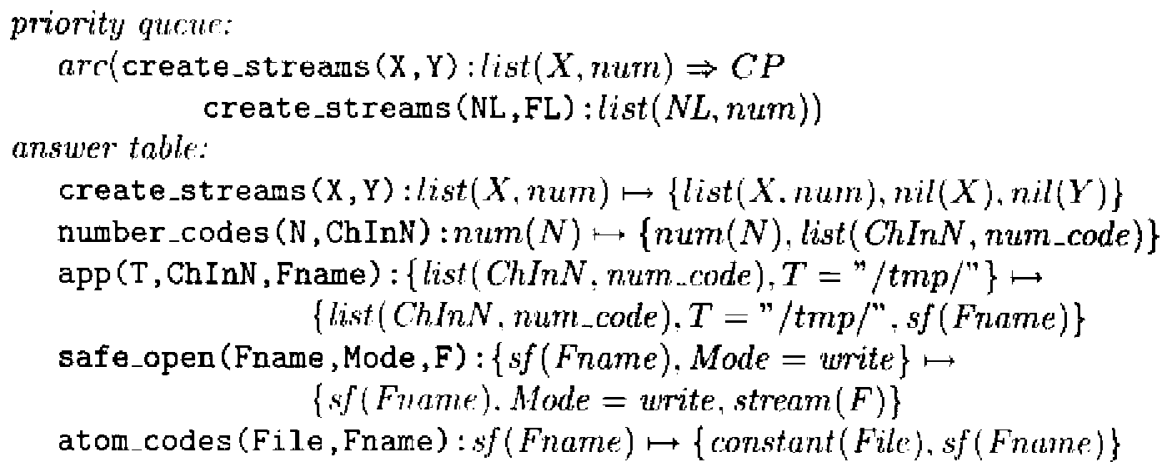




$$
\begin{array}{r}
\text { open (File, Mode, } \mathrm{F}):\{\text { constant }(\text { File }), \text { Mode }=\text { write }\} \\
\{\text { constant }(\text { File }), \text { Mode }=\text { write, } \operatorname{stream}(F)\}
\end{array}
$$

where

$$
\begin{aligned}
& C P=[\operatorname{list}(X, n u m), \operatorname{num}(N), \operatorname{list}(N L, n u m), Y=[F \mid F L] \text {. } \\
& \text { list (ChInN, num_code), sf(Fname), constant (File). } \\
& \text { Mode }=\text { urite, stream }(F), T=" / \operatorname{tmp} / "
\end{aligned}
$$

It is interesting to note that CiaoPP creates the anxiliary type:

$$
\text { sf ("/tmp/" ||A):-list(A, num_code). }
$$

to represent strings which start with the prefix "/tmp/" and continue with a list of type num_code. Since all num_codes are also alphanum_codes. it is clear that. sf $\sqsubseteq$ safe name. This will allow our system to infer that calls to open performed within tiis program satisfy the simple safety policy discussed in Example 4.1. Therefore, the information stored in the answer table is sufficient to attest the safety policy. Also, we use the notation $V a r=$ constant to denote that the system generates a new type whose only element is this constant. as it happens for write. in the entries for safe_open and open and. for "/tmp/", in the cutry lor app.

Now, the call get_answer for the recursive call create_streams (NL, FL): list(NL. num) is made. The answer table is looked up to find the answer and, appropriately ronamed and restricted to the variables in the call, gives $A P_{0}=\{\operatorname{nil}(N L), n i l(F L)$, list $(N L, n u m)\}$. This description is extended to all variables (no change) and then conjoined with $C P$ to give the next annotation $\{\operatorname{mil}(X), \operatorname{nil}(Y), \operatorname{list}(X, n u m)$. list $(Y$, stream $)\}$. We take the least upper bound of this answer with the old answer in the table, giving \{list $(X$. num $)$. hist $(Y$. stream $)\}$. The answer table will replace the surrent amotation for create_streams $(X, Y): \operatorname{list}(X, n u m)$ by:

create_streams $(X, Y): \operatorname{list}(X, n u m) \mapsto\{\operatorname{list}(X, n u m)$,list $(Y$, stream $)\}$ adding the processed are to the dependency are table.

As the answer has changed, an updated event is added to the priority queut. The priority queue contains:

$$
\text { updated(create_streams }(\mathrm{X}, \mathrm{Y}): \operatorname{list}(X . \operatorname{mum}))
$$

The updated event is processed by looking in the dependency arc table for all arcs which have a body literal which is a variant of create_streams $(X, Y): \operatorname{list}(X$, mum) and adding these ares to the priority quene to be reprocessed. There is only one (the last processed arc). After reprocessing this arc we obtain as answer $\{$ list $(X, n u m)$.list $(Y$, stream $)\}$. Taking the least upper bound of this with the old answer, the result is identical to the old answer. henes no updated event is added to the priority quene. As there are no events on the priority quene the inalysis terminates.

As a result of the whole analysis, the answer table computed by CiaoPP contains (anong others) these entries: 


\begin{tabular}{|c|c|c|}
\hline Procedure & Calling Pattern & Success Patteru \\
\hline create_streams $(A, B)$ & list $(A, \pi \mathrm{um})$ & list (A, num),list(B, atream) \\
\hline number_codes $(A, B)$ & $\operatorname{num}(\mathbf{A})$ & $\operatorname{num}(A)$, list $(B, \operatorname{sum}$ _code $)$ \\
\hline$\overline{\operatorname{app}}(\bar{A}, \bar{B}, \bar{C})$ & $\begin{array}{c}A=" 1 / \text { tmp } / ", \\
\text { iist (B, num_code) }\end{array}$ & $\begin{array}{c}A=" / \operatorname{tmp} / ", \\
\text { list }(B, \text { num_code }), \text { sf (C) }\end{array}$ \\
\hline affe_open $(A, B, C)$ & sf $(A), B=$ write & $\operatorname{af}(A), B=$ write, stream (C) \\
\hline atom_codes $(A, B)$ & sf (B) & constant $(A)$, sf $(B)$ \\
\hline open $(A, B, C)$ & constant $(A), B=$ write & constant $(A), B=w r i t e$, stream (C) \\
\hline
\end{tabular}

We show in the next section that the information stored in the above table can be taken as $\operatorname{Cert}_{\alpha x}$, i.e., $\operatorname{Cet}_{\alpha x}=A T$, because it is sufficient to certify that the mobile code is safe according to the policy defined in Example 4.1.

In order to increase accuracy, analyzers are usually multivariant on cals (see, e.g. ${ }^{22)}$ ). Indeed, though not visible in this example, CiaoPP incorporates a multivariant analysis, i.e., more than one triple $\left\langle A: C P_{1} \rightarrow A P_{1}\right\rangle, \ldots,\langle A$ : $\left.C P_{n} \mapsto A P_{n}\right\rangle, n>1$ with $C P_{i} \neq A P_{i}$ for some $i, j$ may be computed for the same procedure descriptor $A$.

\section{\$6 The Verification Condition}

As part of the certification process carried out by the code producer, the verification condition generator (VCGen in Fig. 1) extracts, from the initial assertions $I_{a}$ and the abstraction Cert $t_{c}$ a Verification Condition (VC) which can be proved only if the execution of the code does not violate the safety policy. In particular, we are interested in studying the implications of comparing the intended safety policy described in Section 4, denoted $J_{\alpha}$. with the program abstraction described in Scction 5 , denoted $\llbracket P \rrbracket_{\alpha}$. Therefore. VCGen generates a VC which encodes the comparison $\| P \rrbracket_{\alpha} \Gamma_{\alpha}$ in Equation (2). If VC can be proved (marked as OK in Fig. 1), then the certificate (i.e., the abstraction) is sent together with the program $P$ to the code consumer.

\section{Definition 6.1 ( $V \mathrm{C}$ - verification condition)}

Let $A T$ be an analysis answer table computed for a program $P$ and a set of calling patterns $S$ in the abstract domain $D_{\alpha}$. Let $I$ be an assertion. Then, the verification condition, $V C(I, A T)$, for $I$ w.r.t. $A T$ is defined as follows:

$$
V C(I, A T)::=\left\{\begin{array}{cc}
\bigwedge_{\langle A: C P \mapsto A P\rangle \in A T} & \left(\rho(C P) \sqsubseteq \lambda_{\text {Prec }}^{l} \vee \ldots \vee \rho(C P) \sqsubseteq \lambda_{\text {Prec }}^{n}\right) \\
\bigwedge_{\langle A: C P \mapsto A P) \in A T} & \rho(C P) \sqcap \lambda_{\text {Prec }}=\perp \vee \rho(A P) \sqsubseteq \lambda_{\text {Post }} \\
\text { if } I=\operatorname{calls}\left(B,\left\{\lambda_{\text {Prec }}^{1} ; \ldots ; \lambda_{\text {rec }}^{n}\right\}\right) \\
\text { if } I=\operatorname{success}\left(B, \lambda_{\text {Prec }}, \lambda_{\text {Post }}\right)
\end{array}\right.
$$

where $\rho$ is a variable renaming substitution of $A$ w.r.t. $B$.

If $I_{\alpha}$ is a finite set of assertions, then its verification condition, $V C\left(I_{\alpha}, A T\right)$, is the conjunction of the verification conditions of the elements of $I_{\alpha}$.

Roughly speaking, the VC generated according to Def. 6.1 is a conjunction of boolean expressions (possibly containing disjunctions) whose validity en- 
sures the consistency of a set of assertions w.r.t. the answer table computed by Analysis, see Equation (1). It distinguishes two different cases depending on the kind of assertion. For calls assertions, the VC requires that at least one precondition $\lambda_{\text {Prec }}^{i}$ be a safe approximation of each existing abstract calling patterns for the literal $B$. In the case of success assertions, there are two cases for them to hold. The first one indicates that the precondition is never satisfied and, thus, the assertion trivially holds (and the postcondition does not need to be tested). The second corresponds to the case in which the success substitutions computed by analysis for the procedure are equal or more particular than the one required by the assertion.

\section{Example 6.1 (Verification Condition)}

Consider the answer table generated in Example 5.2 and the calls and success assertions of Fig. 2. According to Definition 6.1, the VC is:

$$
\begin{aligned}
& \operatorname{num}(X) \sqsubseteq \quad(\operatorname{num}(X) ; \operatorname{list}(Y, \text { num_code })) \wedge \\
& \operatorname{sf}(Y) \sqsubseteq \text { (constant(X): string(Y)) } A \\
& \text { constant }(X), Y=\text { write } \sqsubseteq \operatorname{constant}(X) \text {.iomode }(Y) \wedge \\
& \operatorname{sf}(X)[\text { safe_name }(X)
\end{aligned}
$$

Each conjunct corresponds to a calls assertion in Fig. 2 in the same order they appear there. As already mentioned, success assertions for predefined procedures are verified beforehand.

The validity of the whole conjunction can be easily proved by taking into account the following (trivial) relations between the elements in the donain:

$$
\begin{array}{rll}
\operatorname{sf}(X) & \sqsubseteq & \operatorname{string}(X) \\
X=\text { write } & \sqsubseteq & \text { iomode }(X)
\end{array}
$$

Note that the first two conjuncts contain a disjunction in the right hand condition. In the second one, the condition $s f(Y) \sqsubseteq$ (constant $(X)$ : string $(Y))$ holds because $\mathbf{s} f(\mathrm{Y}) \sqsubseteq \operatorname{string}(\mathrm{Y})$.

Therefore, upon creating the answer table and generating the $\mathrm{VC}$, the validity of the whole boolean condition is checked by resolving each conjunct separately. Note that each conjunct consists of comparisons of pairs of abstract substitutions, which simply return either true or false but do not compute any substitution. This validation may yield three different possible outcones: i) the $\mathrm{VC}$ is incleed checked and the answer table is considered a valid abstraction (narked as OK), ii) it is disproved, and thus the certificate is not valid and the code is definitely not safe to run (we should obviously correct the program before contimuing the process); iii) it can neither be proved nor disproved. The latter case happens because some properties are undecidable and the analyzer performs approximations in order to always terminate. Therefore, it may not be able to infer precise enough information to verify the conditions. The user can then provide a more refined description of initial calling patterns or choose a different. finer-grained, domain. Althongh, it is not shown in the picture, in both the ii) and iii) cases, the certification process needs to be restarted until achieving a VC which meets i). 
The following theoren states the soundness of the $\mathrm{VC}$. Intuitively, it amounts to saying that if the $\mathrm{VC}$ holds. then the execution of the program will preserve all safety assertions. Following the notation of. ${ }^{3 / 1}$ we write $\triangle V C$ when $V C$ is valid.

\section{Theorem 6.1 (Soundness of the Verification Condition)}

Let $A T$ be an andysis answer talble for a progran $P$ and a set of calling patterns $S$ in an abstiact domain $D_{\alpha}$ (as defined in Fig. 4). Let $I_{\alpha}$ be a set of assertions. Let $V C\left(I_{c}, A T\right)$ be the verification condition for $I_{\alpha}$ w.r.t. $A T$ (generated as stated in Def. 6.1). If $\triangleright V C\left(I_{\alpha}, A T\right)$, then $P$ satisfies all assertions in $I_{\alpha}$ for all computations described by $S$.

This result directly derives from the fact that the static analysis algorithm computes a safe approximation of the states reached during computation (see Theorem 5.1).

\section{$\$ 7$ Checking Safety in the Consumer}

The checking process performed by the consumer is illustrated on the right hand side of Fig. 1. Initially, the supplier sends the program $P$ together with the certificate to the consumer. To retain the safety guarantees, the consumer can provide a new set of assertions, denoted $I_{\alpha}^{\prime}$, which specifies the Safety Policy required by this particular consumer. It should be noted that $A C C$ is very flexible in that it allows different implementations on the way the safety policy is provided. Clearly, the same assertions used by the producer, denoted $I_{\alpha}$, can be sent to the consumer. But, more interestingly, the consumer can decide to impose a weaker safety condition, i.e., $I_{\alpha x} \sqsubseteq I_{\alpha}^{\prime}$, which can be proved with the submitted abstraction since $\llbracket P \rrbracket_{\alpha} \sqsubseteq I_{\alpha x}$. Also, the imposed safety condition can be stronger, i.e., $I_{a}^{\prime} \sqsubseteq I_{\alpha}$ and it may not be provable if it is not implied by the current abstraction $\llbracket P \rrbracket_{\alpha}$ (which means that the code would be rejected). From the: provided assertions, the consumer must generate again a trustworthy $V C$ and use the incoming certificate to efficiently check that the VC holds. Thus, in the validation process. a code consumer not only checks the validity of the answer table (Equation 3) but it also (re-)generates a trustworthy VC (Equation 4). The validation of $A T$ in Equation 3 is carried out by the Analysis Checker. The re-generation of $V C$ in Equation 4 (and its corresponding validation) is identical to the process already discussed in the previous section. Therefore. this section describes only the first part of the validation process.

\subsection{Fixed-point Checking}

Although global analysis is now routinely used as a practical tool, it is still unacceptable to rum the whole Analysis to validate the certificate since it involves considerable cost. One of the main reasons is that the analysis algorithm is an iterative process which often computes answers (repeatedly) for the same call due to possible updates introduced by further iterations. At each iteration, the algorithm has to manipulate rather complex data structures-which involve performing updates, lookups, etc.-until the fixed point is reached. The whole 
valiclation process is centered around the following observation:

The checking algorithm can be defined as a very simplified "one-pass" analyzer.

The Analysis process can be understood as: Analysis = firedpoint(amalysis_step). I.e., a process which repeatedly performs a traversal of the analysis graph (denoted by analysis_step) until the computed information does not change. The idea is that the simple, non-iterative, analysis_step process can play the role of abstract interpretation-based checker (or simply analysis checker). In other words, check $\equiv$ analysis_step. Intuitively, since the certification process should provide a correct fixed-point result (i.e.. $\left[P \rrbracket_{\Omega}\right.$ ) as certificate, an additiona] analysis pass over this fixed point should not change the result. Otherwise, the current answer table is not a valid abstraction of the program. Thus, in our context, as long as the answer table is valid, one single execution of analysis step is required to validate the certificate, as stated in Equation (3).

\subsection{The Checking Algorithm}

The next definition presents our abstract interpretation-based cheching algorithm. It receives as an additional input a Cert ${ }_{\alpha}$ (which is the analysis fixed point). In a single traversal, it constructs a program analysis graph by using the information in Cert $_{\alpha}$. The algorithm is devised as a graph traversal procedure which places entries in a local answer table, $A T$, as new nodes in the program analysis graph are encountered. Thus, it handles two distinct answer tables: the local $A T+$ the incoming $\operatorname{Cert}_{\alpha}$. The final goal of the checking is to reconstruct the analysis graph and compare the results with the information stored in Cert $_{\alpha}$. As long as Cert $\alpha$ is valid. both results coincide and, thus, the certificate is guaranted to be valid w.r.t. the program.

\section{Definition 7.1 (Analysis Checker)}

Let $P$ br a normalized program and $S$ be a set of calling patterns in the abstract domain $D_{\alpha}$. Let Cert $t_{\alpha}$ be an answer table (or safety certificate) as define'd in Figure 4. The validation of Cert ${ }_{\alpha}$ is performed by the procedure check depicted in Fig. 5. The algorithm uses a local answer table, $A T$, to compute the results (initially it does not contain any entry).

Following the presentation of the analysis algorithm in Section 5.2, we assume that the program $P$ and the answer table are global parameters throughout. the algorithm. The checking algorithm proceds as follows: as in the analysis algorithm. the procedure process_arc is aimed at computing the resulting description $C P_{a}$ after processing a given literal $B_{k, i}$. The computed result is used to process the next literal in the rule when $B_{k, i}$ is not the last one. Otherwise, the computed result constitutes indeed the computed answer for the rule. The difference w.r.t. the analyzer is that the answer is combined with the corresponding answer supplied by the certification process in Cert . If $_{\text {Cert }}$ is valid, the comparison stould hold; otherwise the process prompts an error and the program is not safe to run. Therefore, no control structure is needed in order to guarantee that a fixed point is reached. This eliminates the noed for the "event. 


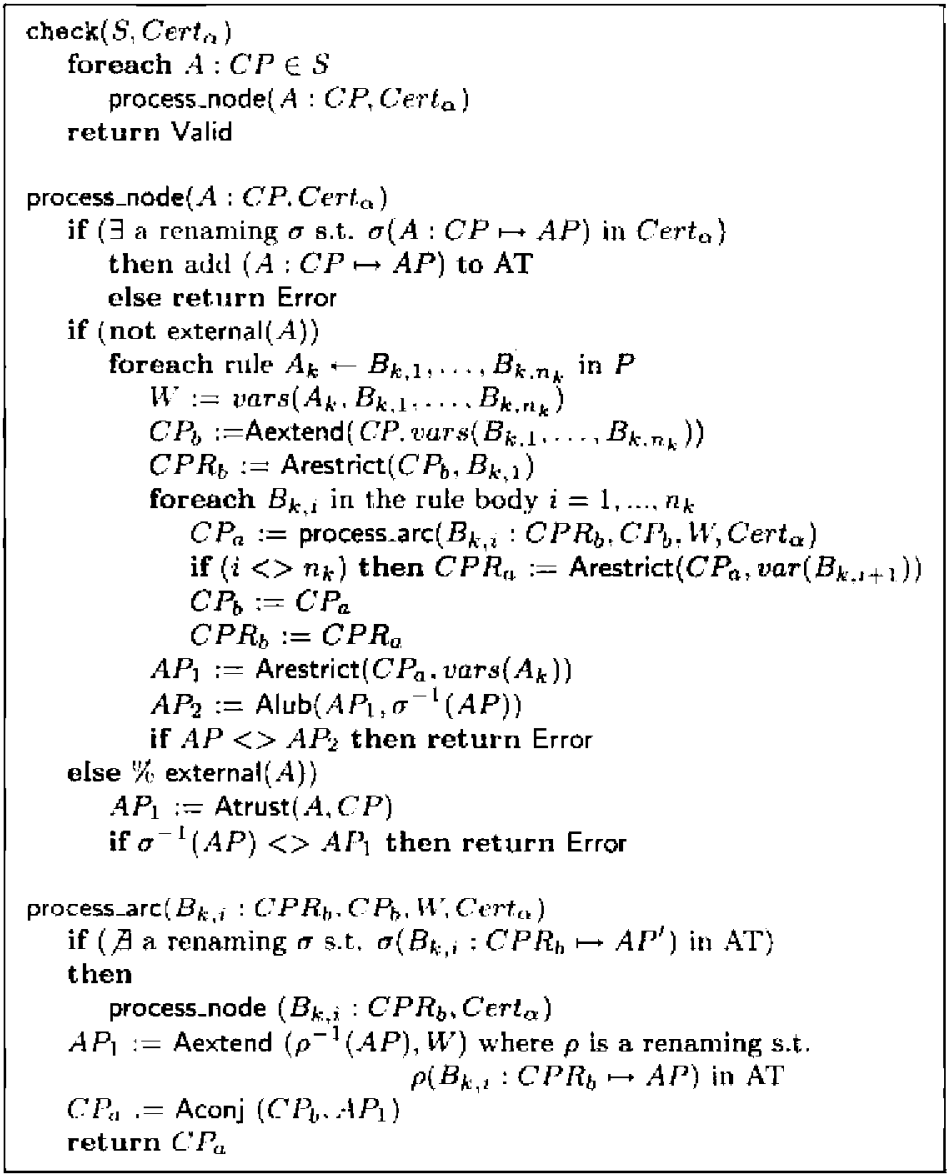

Fig. 5 Abstract Interpretation-based Checking in CiaoPP

queue" of the analysis algorithm in Fig. 4. Moreover, since only one traversal of the analysis graph is to be performed, no detailed dependency information is required. This eliminates the need for the "dependency arc table" of the analysis algorithm. As a result, check is a suitable procedure for determining the validity of the certificate.

The following theorem ensures that algorithm check is able to validate safety certificates which are stored in a valid analysis answer table.

\section{Theorem 7.1 (partial correctness)}

Let $P$ be a progran, let $S$ be a set of calling patterns in an abstract domain $D_{\alpha}$. Let $\operatorname{Cert}_{\alpha}$ be an answer table for $P$ and $S$ as defined in Fig. 4. Then, check $\left(S\right.$, Cert $\left._{\alpha}\right)$ terminates and, if it returns Valid, then Cert $_{\alpha}$ is an abstraction of $P$ and $S$.

The theorem is implied by the definition of fixed point and the fact that check is a single pass of a correct Analysis algoritlmu. ${ }^{22}$ Indeed. it is immediate to 
see that algorithm check has been obtained as a simplification of the algorithnn Analysis.

Another issue is the efficiency of the checking algorithm. Our point to justify an efficient behavior of check for validating an answer table is that it performs a single graph traversal. Indeed, for a regular type domain, ${ }^{12}$ demonstrates that directional type-checking for logic programs is fixed-parameter linear. Section 8 reports experimental evidence of efficiency issues.

\subsection{An Example}

We describe the more representative steps that algoritim check performs in order to validate the answer table of Example 5.2 .

\section{Example 7.1}

Consider the answer table, called Cert $_{\alpha}$. of Example 5.2. First, procedure process node looks up an answer for the initial calling jattern in Certa and arlds the entry

(create_streams $(\mathrm{X}, \mathrm{Y}): \operatorname{list}(X$, num $) \mapsto A P=\{\operatorname{list}(X$, num $)$, list $(Y$, stream $)\}\rangle$

to the answer table $A T$ (note that, for short, we use $A P$ to denote this particular answer pattern). Since there are two rules defining create_streams the outermost loop performs two iterations:

Iter 1. We start by describing the processing of the first rule (although the order is irrelevant). Since the first literal $x=[]$ in the rule body is a comstraint, its description is computed within procedure process_arc by adding its alsstract description. i.e.. $\{n i l(X)\}$, to the initial description $\{l i s t(X, n u m)\}$, resulting in $\{n i l(X), \operatorname{list}(X, n u m)\}$. Similarly, the analysis for the second constraint adds $\{n i l(Y)\}$ to the former description producing $\{n i l(X)$, nil $(Y)$. lsst $(X, n u m)\}$. Upon exiting the innermost loop, the disjunction of this description with the answer stored in Cert $\alpha$ is calculated:

$$
A P_{2}:=A l u b(\{\operatorname{nil}(X), \operatorname{nil}(Y), \operatorname{list}(X, \operatorname{num})\}, A P)
$$

since $\operatorname{nil}(X) \sqsubseteq \operatorname{list}(X, n u m)$ and $\operatorname{nil}(Y) \sqsubseteq \operatorname{list}(Y$, stream $)$, then $A P_{2}=A P$. Thus, the certificate holds for this rulc.

Iter 2. In the second iteration, we find eight literals in the rule boty. Thus, the innermost loop performs the following ciglit steps. The first two traversals deal with the constraints for $X$ and $Y$, and are similar to Iter 1 . They produce the calling patteriı

$$
\{\operatorname{list}(X, n u m), \operatorname{num}(N) . \operatorname{list}(N L . n u m) . Y=[F \mid F L]\}
$$

The next literal, number_codes, in the rule body is an external procedure. thus, process_node uses the parametric routine Atrust which gives the answer $\{n u m(N)$, list $(C h I n N$, num_code $)\}$ for it. This answer is conjoined with the description of the program point immediately before the literal, i.e.:

$\{$ list. $(X, n u m), \operatorname{num}(N)$, list $(N L, n u m), Y=[F \mid F L]$, list $(C h I n N$, num_code $)\}$ 
The remaining intermediate literals are dealt in a similar way (see Example 5.2 for more specific details). Let us just consider the processing of the recursive call to create_streams, for which we get as final description:

$$
\begin{aligned}
C P=[ & \operatorname{list}\left(X, \operatorname{num}_{1}\right), \operatorname{mum}(N), \operatorname{list}(N L, \text { num }), Y=[F \mid F L], \\
& \text { list }(\text { ChInN, num_code }), \text { sf }(\text { Frame }), \text { constant }(\text { File }), \text { Mode = write, } \\
& \text { stream }(F), T=" / \text { tmp } / "]]
\end{aligned}
$$

Now, process node finds out that $A T$ already contains an answer pattern for this procedure. Then, both calling patterns are conjoined: $C P_{a}:=A c o n j(C P, A P)$ and restricted to variables $\mathrm{X}$ and $\mathrm{Y}$, obtaining $C P_{a}=A P$ as final result. Upon retırn from process_arc, it performs the disjunction of the computed answer with the answer supplied by $\operatorname{Cert}_{\alpha}: \quad A P_{g}:=\operatorname{Aluh}\left(C P_{n}, A P\right)$. Since $C P_{a}=A P$ and also the result, $A P_{2}=A P$, coincides with the one in the certificate. the proof is velidated and the algorithm terminates in a single graph traversal for the initial query. Note that in the analysis example, there is an additional full iteration due to the existence of update events which make the analyzer re-process all arcs which depend on a calling pattern whose answer has been updated. It is well knowt that several passes over the program are often needed to reach a fixed point.

\section{$\$ 8$ Experimental Results}

In this section we show some experimental results aimed at studying two crucial points for the practicality of our proposal: the checking time as compared to the analysis time, and the size of certificates. We have implemented the checker as a simplification of the generic abstract interpretation system of CiaoPP. It shonld be noted that this is an efficient, highly optimized, state-ofthe-art analysis system which is part of a working compiler. Both the analysis and checker are parametric w.r.t. the abstract domain. In these experiments they both use the same implementation of the donain-dependent functions of the sharing +freeness domain. ${ }^{321}$ We have selected this domain because the information it infers is very useful for reasoning about instantiation errors, which is a crucial aspect for the safety of logic programs. The whole system is implemented in Ciao 1.11\#200 $0^{21}$ with compilation to bytecode. All of our experiments have been performed on a Pentium 4 at $2.4 \mathrm{GHz}$ and 512MB RAM running GNU Linux RH9,0. The Linux kernel used is 2.4 .25 , customized with the hrtime patch to provide improved precision and resolution in time measurements.

\subsection{Checking Time}

Table 1 presents our experimental results regarding checking time. Execution times are given in milliseconds and measure runtime. They are computed as the arithmetic mean of five runs. A relatively wide range of programs has been used as benchmarks. They are the same ones used in ${ }^{22)}$ where they are described iu some detail. For each benchnark, the columns for Analysis are the following: $\mathrm{P}_{A}$ is the time required by the preprocessing phase, in which program rules are processed and stored in the format required by the analyzer. The analysis time 
Table 1 Checking Time

\begin{tabular}{|c|c|c|c|c|c|c|c|c|}
\hline & \multicolumn{3}{|c|}{ Analysis } & \multicolumn{3}{|c|}{ Checking } & \multicolumn{2}{|c|}{ Sptedup } \\
\hline Bench & $P_{A}$ & $\mathrm{An}$ & $T_{A}$ & $\mathbf{P}_{C}$ & $\mathrm{Ch}$ & $\mathrm{T}_{C}$ & $A / C$ & $\mathrm{~T}_{A} / \mathrm{T} \mathrm{T}_{C}$ \\
\hline EiakJ & 2 & 87 & 89 & 2 & 71 & 72 & $\overline{1 . \overline{2}}$ & 1.2 \\
\hline $\operatorname{ann}$ & 22 & 452 & 474 & 1.8 & 254 & 272 & 1.8 & 1.7 \\
\hline bid & 4 & 56 & $6 \overline{0}$ & 4 & 35 & 38 & 1.6 & 1.6 \\
\hline boyer & 9 & 143 & $15 \overline{1}$ & 7 & 85 & 92 & $\overline{1.7}$ & 1.6 \\
\hline browse & $\overline{3}$ & 14 & 17 & 3 & 12 & 15 & 1.2 & 1.2 \\
\hline deriv & 2 & 86 & $\overline{88}$ & 1 & 19 & 20 & 4.6 & 4.4 \\
\hline gramitiar & 2 & 10 & 12 & 2 & 9 & 21 & 1.1 & $1+1$ \\
\hline hanoiapp & 2 & 25 & 26 & 2 & 16 & 58 & 1.5 & 1.5 \\
\hline mmatrix & 1 & $\overline{13}$ & 14 & $\bar{I}$ & 10 & 11 & 1.3 & 1.3 \\
\hline occur & $\overline{2}$ & 16 & 18 & 2 & 10 & 12 & 1.7 & 1.6 \\
\hline progeon & $\overline{2}$ & 13 & 15 & 2 & 9 & 11 & {$[5$} & 1.4 \\
\hline read & $\overline{9}$ & 792 & 801 & 8 & 488 & 497 & 1.6 & 1.6 \\
\hline gplan & 13 & 1411 & 1424 & 11 & 962 & 973 & 1.5 & 1.5 \\
\hline qsortapp & 1 & 20 & 21 & 1 & 12 & 14 & 1.6 & 1.5 \\
\hline query & 5 & 11 & 15 & 4 & 9 & 12 & 1.2 & 1.3 \\
\hline rdtok & 8 & 141 & 449 & 6 & 43 & 49 & 3.3 & 3.1 \\
\hline serialize & 2 & 40 & 42 & 2 & 17 & 19 & 2.3 & 2.2 \\
\hline warplatn & 8 & $17 \overline{3}$ & $\overline{181}$ & 7 & $16 \%$ & 115 & 1.6 & 1.6 \\
\hline witt & 16 & 196 & 212 & 14 & 72 & 86 & 2.7 & $2 . \overline{5}$ \\
\hline zebra & 3 & 94 & $\sqrt{17}$ & 3 & 00 & 92 & 1.1 & 1.0 \\
\hline Overall & & & & & & & 1.63 & 1.61 \\
\hline
\end{tabular}

proper is shown in columm An. The actulal time needed for analysis the sum of these two times- is shown in column ' $T_{4}$. Similarly, in the case of checking, three columus are shown. The preprocessing phase, $\mathrm{P}_{C}$, includes asserting the certificate in addition to asserting the program to be analyzed. As the figures show, the overhead required for asserting the certificate is negligible. Colum $\mathrm{Ch}$ is the time for executing the checking algoritlm. Finally, $\mathrm{T}_{C}$ is the total time for checking. The columns under Speedup compare analysis and checking times. As can be seen in columns $A / C$ and $T_{A} / T_{C}$. the checking algorithm is faster than the analysis algorithm in all cases. The actual speedup ranges from almost none, as in the case of zebra, to over four times faster in the case of deriv. The last row summarizes the results for the different benchmarks using a weighted mean. which places more importance on those benchmarks with relatively larger analysis times. We use as weight for each program its actual analysis time. We believe that this weighted mean is more informative than the arithmetic mean, as, for example. doubling the speed in which a large and complex program is analyzed (checked) is more relevant than achieving this for small. simple programs. Overall, the speedup is 1.63 in just analysis time, or 1.61 if we also take into account the preprocessing time. We believe that the achieved speedup is significant taking into account that CiaoPP's analyzer for this domain is highly optimized and converges very efficiently. ${ }^{391}$ However, it is to be expected that, for other domains and implementations, the relative gains will be higher.

\subsection{Certificate Size}

Table 2 shows our cxperimental results regarding certificate size, coded in compact (fastread) format, for the different benchmarks and compares it to the size of the source code for the same program and to the size of the corresponding 
Table 2 Certificate Sizo

\begin{tabular}{|c|c|c|c|c|c|}
\hline & Sesuree & \multicolumn{2}{|c|}{ Byte Code } & \multicolumn{2}{|c|}{ Cartificate } \\
\hline Bench & Source & ByteC & $\overline{\mathrm{B} / \mathrm{S}}$ & Cert & $0 / 5$ \\
\hline fliakl & $15 \overline{55}$ & 3805 & 2.4 & 3090 & 2.0 \\
\hline antr & 127.45 & 43884 & 3.4 & $2 \overline{4475}$ & 1.9 \\
\hline bid & 4945 & 10376 & 2.1 & 5939 & 1.2 \\
\hline boyer & 11010 & 32522 & 3.0 & 12300 & 1.1 \\
\hline browse & 2589 & 8467 & 3.3 & $166 \mathrm{I}$ & 0.6 \\
\hline deriv & 957 & 4221 & 4.4 & 288 & 0.3 \\
\hline gsйm & 1598 & 3182 & 2.0 & 1259 & 0.8 \\
\hline Franoiape & 1172 & 2264 & 1.9 & 2325 & 2.0 \\
\hline mutuatrix & 557 & 1053 & 1.9 & 880 & 1.6 \\
\hline occur & 1367 & 6903 & 5.0 & 1008 & 01.6 \\
\hline progeom & 1619 & 3570 & 2.2 & 2148 & 1.3 \\
\hline read & 11843 & 24619 & 2.1 & $25 \overline{359}$ & 2.1 \\
\hline qplan & 9983 & 33472 & 3.4 & 20509 & 2.1 \\
\hline qsortapp & 664 & 1176 & 1.8 & 2355 & 3.5 \\
\hline quety & 2090 & 8833 & 4.2 & 531 & 0.3 \\
\hline lditok & 13704 & 15354 & 1.1 & 6533 & 0.5 \\
\hline setialize & 987 & 3801 & 3.9 & 1779 & 1.8 \\
\hline warplan & 3203 & 23971 & 4.6 & 15.305 & $2 . \overline{9}$ \\
\hline wit.t & 17681 & 41760 & 2,4 & 10131 & 1.1 \\
\hline zebra & $-2 \overline{284}$ & 5396 & 2.4 & 4058 & 1.8 \\
\hline Overall & 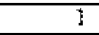 & & 2.66 & & $\overline{1.44}$ \\
\hline
\end{tabular}

bytecode. To make this compurison fair, we subtract 4180 bytes from the size of the bytecode for each program: the size of the bytecode for an empty program in this version of Ciao (minimal top-level drivers and exception landlers for any executable). The results show the size of the certificate to be quite reasonable. It. rarlges from 0.3 times the size of the source code (for deriv) to 3.5 (in the case of qsortapp). Overall, it is 1.44 times the size of the source code. We consider this acceptable since in general (C)LP programs are quite compact (up to 10 times more compact than equivalent imperative programs). In fact, the size of source plus certificate is smaller $(1+1,44)$ than that of the bytecode $(2.66)$.

\section{\$9 Conclusions and Related Work}

We have presented abstraction-carrying code (ACC) as a novel enabling technology for PCC, which follows the standard strategy of associating safety certificates to programs but it is based throughout on the use of abstract interpretation techniques. We argue that $\mathrm{ACC}$ is highly flexible one aspect being the parametricity on the abstract clomain inherited from analysis engines as exemplified by those used in (C)LP. We argue that our proposal brings the expressiveness, flexibility, and antomation which is inherent in the abstract interpretation techniques developed in logic programming to this area. Our approach has been illustrated by using the CiaoPP system. This system already uses a combination of abstract interpretation, abstract specialization, and a fiexible assertion language, to perform program debugging, verification, and optimization with a wide variety of domains. Other approaches to abstract verification and debutgging have also been proposed (see ${ }^{15.21)}$ for further references). The system las been enhanced to produce certificates as dictated by the ACC scheme, as an integral part of the static debugging and verification performed during the 
program development process. A simplified version of the analysis framework of CiaoPP has also been developed that serves as an efficient checker of the certificates. The approach is currently being tested in a number of pervasive applications using au embedded version of the Ciao system which runs on PDAs and Gumstix processors. Ongoing work also includes the study of techuiques for further reducing the size of certificates," and reducing the checking time.

It is important to note that our approach will wo:k directly in otber programming paradigms, such as imperative or functional programming (the latter already covered in our current system, since Ciao supports functional programming), as long as a static arlalyzer/checker is available. Note that the fundamental components of the approach (fixed-point semantics and abstract interpretation) have both been widely applied also in these paradigms. In fact, analyzers have been recently developed, for example, for analysis and verification of Java, which are direct adaptations of essentially the same parametric fixpointbased analysis algorithms that we have used in this work on the producer side. ${ }^{2.1}$ The ACC approach is thus directly applicable in this context.

Our approach differs from existing approaches to PCC in several aspects. In our case, the certificate is computed antomatically on the producer side by an abstract interpretation-based analyzer and the certificate takes the form of a particular subset of the analysis results. The burden on the consumer side is reduced by using a simple one-traversal checker. which is a very simplified and efficient abstract interpreter which does not need to compute a fixed point.

A type-level dataflow analysis of Java virtual machine brtecode is also the basis of several existing verifiers. ${ }^{26.27)}$ and some are loosely based on abstract interpretation. These analyses allow proving that the program is correct w.r.t. type-related correctness conditions. $\operatorname{In}^{401}$ a proposal is presented to split the type-based bytecode verification of the KVM (an embedded variant of the JVM) in two phisses, where the producer first computes the certificate by means of a type-based dataflow analyzer and then the consumer simply checks that the types provided in the code certificate are valid. As in our case, the second phase can be done in a single, linear pass over the bytecode. However, these approaches are limited to types.

Let us note that our checker is part of the trusted computing base (TCB) and. hence, the code consumer has to trust also the domain operations. Other approaches to PCC use logic-based verification methods as enabling technology, an example is ${ }^{45)}$ which formalizes a simple assembly language with procedures and presents a safety policy for arithmetic overflow in Isabelle/HOL. Recently, a PCC architecture based on Certified Abstract Interpretation ${ }^{11}$ has been proposed by Besson et al." This proposal follows the basics of ACC for certificate generation and checking, but relies on a certified checker specified in $\operatorname{Coq}^{53}$ in order to reduce the TCB. In contrast to our framework, this work is restricted to safety properties which hold for all states and, for now, it has only been implemented for a particular abstract domain.

The coexistence of several abstract domains in our framework is somewhat related to the notion of models to capture the security-relevant properties of code, 
as addressed in the work on Model-Carrying Code (MCC). ${ }^{121} \mathrm{MCC}$ enables code consumers to try out different security policies of interest and select one that can be statically proved to be consistent with the model associated to the unt rusted code. However, models are intended to describe low-level properties and their combination has not been studied, which differs from our idea of combining (high-level) abstract domains. Another approach based on model checking (and also types) is that of ${ }^{* 0}$. It is based on sending the "predicate abstraction" used in model checking to help reduce the state space search at the receiving end, where model checking is performed again on the received program. This approach is thus also quite different from ours, although by coincidence (because of the use of a predicate abstraction) it. has been independently given the same name. Perhaps the most obvious difference is that this approach does not exploit the fundamental idea of our proposal of constructing iteratively a fixpoint on the producer's end and checking it without any iteration at the receiving end. Also, it is not parametric on a set of domains as our generic model and it is presented only informally.

As a final consideration, it should be noted that while the particular instance of ACC that we have described in detail is actually defined at the sourcelevel, in most existing PCC franeworks the code supplier typically packages the certificate with the object code rather than with the source code (we assune that both are untrusted). This is without loss of generality because the basic principles of our approach can also be applied to bytecode or machine code. Note that a good ntumber of abstract interpretation-based analyses have been proposed in the literature for bytecode and machine code, most of which compute a fixpoint during analysis which can be checked in one pass at a receiving end using the general principle of our proposal. More concretcly, the work mentioned above which uses similar fixpoint-based analysis algorithus to those that we have applied in this work on the producer side ${ }^{2 \theta}$ performs the analysis and verification directly on Java bytecode, and thus supports the applicability of our approach to bytecode. In fact, also in recent work, even the concrete CLP verifier used in our ACC inplementation (CiaoPP) has itself been shown to also be applicable without modification to Java bytecode via a transformational approach. ${ }^{2}$

In any case, both approaches (ACO for source code and $\mathrm{ACC}$ for object code) are of interest from our point of view: clearly, in many cases the source code is simply not available to the consumer and even when there is a choice between object and source code, using object code means reducing the trusted computing base in the consumer since there is no need for a compiler. On the other hand, open-source code is becoming much more relevant these days (in fact, Ciao and CiaoPP are themselves GNU-licensed and available in source code for reviewing and modification). As a result, it is now realistic to expect that a relatively large amount of untrusted source code is available to the consumer. The advantages of open-source with respect to safety are important since it allows inspecting the code and applying powerful techniques for program analysis and validation which allow inferring information which may be difficult to observe in low-level. compiled code. This allows handling richer properties which in turn potentially 
allow more expressive safety policies.

\section{Acknowledgements}

The authors would like to thank the anonymous referecs for their useful comment.s. This work was funded in part by the Information Society Technologies programme of the European Commission, Future and Emerging Technologies under the IST-15905 MOBIUS integrated project. by the Spanish Ministry of Science and Education under the MEC TIN2005-109207-C03 MERIT project, and by the Region of Madrid under the CAM S-1505/TIC/0407 PROMESAS project. Part of this work was performed during a research stay of Elvira Albert and Germán Puebla at UNM supported by respective grants from the Secretaría de Estado de Educación y Universidades. Spanish Ministry of Science and Education. Manuel Hermenegildo is also supported by the Prince of Asturias Chair in Information Science and Technology at UNM,

\section{References}

1) Albert, E., Arenas, P., Puebla, G. and Hermentegitolo. M., "Reduced Certificates for Abstraction-Carrying Code," in 2end International Conference on Logic: Programming (ICLP 2006). LNCS 4079. Springer-Verlag, pp. 163-178. August 2006.

2) Albert, E.. Gómez-Zamalloa, M.. Hubert. L. and Puebla, G., "Verification of Java Bytecode using Analysis and Transformation of Logic Programs." in Ninth Int. Symp. on Practical Aspects of Declaratine Languages, LNCS 4354, SpringerVerlag, pp 124-139, January 2007.

3) Appel. A. and Felty, A. "Lightweight Lemmas in lambda-Prolog." in Proc. of ICLP'99. MIT Press, pp. 411-425. 1999.

4) Aspinall, D., Gilmore, S., Hofmann. M. Sannella, D. and Stark. I., "Mobile Resource Guarantees for Smart Devices." in CASSIS 04 (Barthe, G.. Burdy, L., Husman. M. Lanet, J.-L. and Muntean, T. eds.), LNCS 3362. Springer, pp. $1-27,2005$.

5) Barras, B., Boutin, S.. Cornes, C., Courant, J., Filliatre. J., Gimenez. E., Herbelin, H., Huet. G., Munoz, C., Murthy: C., Parent. C., Paulin-Mohring. C., Saibi, A. and Werner, B, "The Cog proof assistant reference manual : Version 6.1," Technical Report RT-0203, 1997, citeseer.ist.psu.edu/barras97coq.html.

6) Bernard, A. and Lee, P. "Temporal logic for proof-carrying code," in Proc. of CADE'02. LINCS, Springer, pp. 31-46, 2002.

7) Besson, F., Jensen, T. and Pichardie, D., "A pec architecture based on certified abstract interpretation," in Proc. of First Int. Workshop on Emerging Applications of Abstract Interpretation (EAAI06). Elertronir Notes in Theoretical Computer Science (ENTCS), 2006.

8) Bruynooghe, M, "A Practical Framework for the Alstract Iuterpretation of Logic Programs," Joumal of Logic. Programminty. 10. pp. 91-124. 1991.

9) Bueno. F.. Cabeza., D., Carro. M. Hennenegildo. M. López-Garciá, P. atul Puebla, G.. "Tle Clao Prolog Systom. Reference Mamual (v1.8)." The Ciao 
System. Documentation Scries-TR CLIP4/2002.1. School of Computer Science, Technical University of Madrid (UPM), May 2002. System and on-line version of the manual available at http://www.cianhome.org.

10) Bueno, F.. García de la Banda, M. and Hermenegildo, M., "Effectiveness of Global Analysis in Strict Independence-Based Automatic Program Parallelization," in Int. Symp. on Logic. Programming. MIT Press. pp. 320-336. November 1994.

11) Gachera, D., Jensen. T., Pichardie, D. and Rusu, V., "Extracting a Data Flow Analyser in Constructive Logic," in Proc. of ESOP 2004. LNCS 2986, pp. 385400,2004 .

12) Charatonik, W., "Directional Type Checking for Logic Programs: Beyond Discriminative Types." in Proc. of ESOP 2000. LINCS 1782. pp. 72-87, 2000.

13) Le Charlier, B., Degimbe, O., Michael, L. and Van Hentenryck, P., "Optimization Techniques for General Purpose Fixpoint Algorithms: Practical Efficiency for the Abstract Interpretation of Prolog." in Workshop on Static Analysis. Springer-Verlag, pp. 15-26. September 1993.

14) Le Charlier, B. and Van Hentenryck, P, "Experimental Evaluation of a Generic Abstract Interpretation Algorithm for Prolog." ACM Transactions on Programming Languages and Systems. 16(1), pp. 35-101, 1994.

15) Comini, M., Gori, R., Levi, G. and Volpe, P., "Abstract Interpretation based Verification of Logic Programs," Electr. Notes Theor. Comput. Sci, 30(1), 2000.

16) Cousot, P. and Consot, R., "Abstract Interpretation: a Unified Lattice Model for Static Analysis of Programs by Construction or Approximation of Fixpoints," in Proc. of POPL 77, pp. 238-252, 1977.

17) Dart, P.W. and Zobel. J., "A Regular Type Language for Logic Programs," in Types in Logic Programming, MIT Press, pp. 157-187, 1992.

18) Debray, S.K., "Static Inference of Modes and Data Dependencies in Logic Programs," ACM Transactions on Proyramming Langunges and Systents, 11(3), pp.:418-450, 1989.

19) Debray, S.K.(ed.), "Abstract Interpretation". Journal of Logic Programming. Special 1ssue: Vol. 13(1-2), North-Holland, July 1992.

20) Früwirth,T., Shapiro, E., Vardi, M.Y. and Yarcteni, E., "Logic programs as types for logic programs," in Proc. LICS'91,pp, 300-309, 1991.

21) Hermenegildo, M., Puebla, G., Bueno, F. and López-García, P., "Program Development Using Abstract Interpretation (and The Ciao System Preprocessor)," in Proc. of SAS 03, LINCS 2694, Springer, pp. 127-152, 2003.

22) Hermenegildo, M., Puebla, G., Marriott, K. and Stuckey, P.+ "Incremental Analysis of Constraint Logic Programs," ACM Transactions on Programming Languages and Systems, 22(2), pp. 187-223, Marclı 2000.

23) Hermenegildo, M., Warren, R. and Debray, S.K., "Global Flow Analysis as a Practical Compilation Tool." Journal of Logir Programming. 13(4). pp. 349-367. August 1992.

24) Jaffar, J. and Maher, M.J., "Constraint Logic Programming: A Sturvey," Jourtaal of Logic: Programming, 19/20, pp. 503-581, 1994.

25) Kelly, A., Marriott, K.. Søndergaard, H. and Stuckey, P.J.. "A practical objectoriented analysis engine for CLP," Software: Practice and Experience, 28(2), pp. $188-224,1998$. 
26) Xavier Leroy, "Java bytecode verification: algorithms and formalizations," Journal of Automated Reasoning, 30(3-4), pp. 235-269, 2003.

27) Lindholm. T. and Yellin, F., The Javn Virtual Machine Specification, AddisonWesley, 1907 .

28) Marriott, K., Søndergaard. H. and Jones, N.D.. "Denotational Abstract. Interpretation of Logic Programs," ACM Transactions on Programming Languages and Systems, 16(9), pp. 607-648, 1994.

29) Méndez-Lojo, M., Navas, J. and Hermenegildo, M., "An Efficjent, Parametric Fixpoint Algorithm for Analysis of Java Bytecode," in ETAPS Workshop on Bytecode Semantics, Verification, Analysis and Trartsformation (BYTECODE'07), Electronic Notes in. Theoretical Computer Science. Elsevier - North Holland, March 2007. To appear.

30) Morrisett, G., Walker, D., Crary, K. and Glew. N., "From system F to typed assembly language," ACM Transactions on Programming Languages and Systems, 21(9). pp. 527-568, 1999.

31) Muthukumar, $\mathbb{K}$. and Hermenegildo, M., "Deriving A Fixpoint Computation Algorithm for Top-down Abstract Interpretation of Logic Programs." Technical Report ACT-DC-153-90, Microelectronics and Computer Technology Corporation (MCC), Austin, TX 78759, April 1990.

32) Nuthukumar, K. and Hermenegildo, M., "Combined Determination of Sharing and Freeness of Program Variables Through Abstract Interpretation." in Int. Conf. on Logic Programming. MIT Press, pp. 49-63. June 1991.

33) Muthukunar, K. and Hermenegildo. M., "Compile-time Derivation of Variable Dependency Using Abstract Interpretation," Journal of Logic Programming, 13(2/3). pp.315-347, July 1992.

34) Necula, G., "Proof-Carrying Code," in Proc. of POPL '97, ACM Press, pp. $106-$ $119,1997$.

35) Necula, G. and Lee. P., "The Design and Implementation of a Certifying Conpiler," in Proc. of PLDI98, ACM Press. 1998.

36) Necula. G.C. and Rahul, S.P., "Oracle-based checking of untrusted software." in Proc. of POPL 01. ACM Press. pp. 142-154. 2001.

37) Ptuebla, G., Bueno, F. and Hermenegildo, M., "An Assertion Language for Constraint Logic Programs," in Analysis and Visualization Tols for Constraint. Programming, LINCS 1870, Springer, pp. 23-61, 2000.

38) Puebla, G., Correas, J.. Hermenegildo, M.. Bueno, F., Garcia de la Banda, M., Marriott. K. and Stuckey. P.J.. "A Generic Framework for Context-Sensitive Analysis of Modular Programs," in Program Development in Computational Logic, A Decade of Research Advances in Logic-Based Program Developtnent (Bruynooghe, M. and Lau. K. eds.). LNCS 3049 , Springer-Verlag. Heidelberg. Germany, pp. 234-261. August 2004.

39) Puebla, G. and Hermenegildo, M., "Optimized Algorithms for the Incremental Analysis of Logic Programs," in SAS96, LINCS 1145, Springer, pp. 270-284, 1996.

40) Rose, K., Rose, E., "Lightweight bytecode verification," in OOPSLA Workshop on Formal Underpinnings of Java, 1998.

41) Santos-Costa, V., Warren. D.H.D. and Yang, R., "The Andorra-I Preprocessor: Supporting Full Prolog on the Basic Andorra Model." in Int. Conf. on Logic Programming, MIT Press, pp. 443-456, June 1991. 
42) Sekar, R., Venkatakrishnau, V.V., Bası, S.. Bhatkar, S. and DuVarney, D., "Model-carrying code: A practical approach for safe execution of untrusted applications." in Proc. of SOSP'09. ACM, pp. 15-28, 2003.

43) Van Roy, P. and Despain, A.M., "High-Performance Logic Programming with the Aquarius Prolog Compiler," IEEE Computer Magazine, pp. 54-68, January 1992.

44) Vaucheret, C. and Bueno, F.. "More Precise yet Efficient Type Inference for Logic Programs," in Int. Static Analysis Symp., LNCS 2477, Springer-Verlag, pp. 102-116, September 2002.

45) Wildmoser, M. and Nipkow, T., "Certifying Machine Code Safety: Shallow Versus Deep Embedding," in 17th Int. Conf. on Theorem Proving in Higher Order Logics, LNCS 3233. Springer, 2004.

46) Xia, S. and Hook. .J.: "Experience with Abstraction Carrying Code," in Electronis: Notes on Theo. Comp. Sci., 89, Elsevier, 2003. 